hep-ph/9501336

INFN-FE 01-95

MIT-CTP-2404

\title{
Could the Supersymmetric Higgs Particles Naturally be Pseudo-Goldstone Bosons? ${ }^{1}$
}

\author{
Zurab Berezhiani \\ INFN, Sezione di Ferrara, I-44100, Italy, \\ Institute of Physics, Georgian Academy of Sciences, 380077 Tbilisi, Georgia \\ and \\ Csaba Csáki and Lisa Randall] \\ Center for Theoretical Physics \\ Laboratory for Nuclear Science and Department of Physics \\ Massachusetts Institute of Technology \\ Cambridge, MA 02139, USA
}

\begin{abstract}
The doublet-triplet splitting problem is perhaps the most problematic aspect of supersymmetric grand unified theories. It can be argued that the most natural reason for the Higgs doublets to be light is that they are pseudo-Goldstone bosons associated with the spontaneous breakdown of an accidental global symmetry. In this paper we discuss the possibility of implementing this idea in the SU(6) model of refs. [11, 12, 13, 14]. We show that although it is simple to generate an accidental symmetry of the renormalizable terms of the potential, it is quite difficult to construct a model which allows for the preservation of the accidental symmetry in the nonrenormalizable terms. We summarize the constraints on such models and then give three different ways to construct a superpotential where the dangerous mixing terms are sufficiently suppressed even in the presence of nonrenormalizable operators. With these examples we demonstrate the existence of consistent models implementing the Higgs as pseudo-Goldstone boson scheme. We extend one of the three examples to include fermion masses. We also show that when restricted to regular group embeddings the only possible models without light triplets are trivial generalizations of the $\mathrm{SU}(6)$ model we consider.
\end{abstract}

\footnotetext{
${ }^{1}$ Supported in part by DOE under cooperative agreement \#DE-FC02-94ER40818.

${ }^{2}$ NSF Young Investigator Award, Alfred P. Sloan Foundation Fellowship, DOE Outstanding Junior Investigator Award.
} 


\section{Introduction}

The unification of the gauge couplings of supersymmetric grand unified theories at about $10^{16} \mathrm{GeV}$ is strong evidence that the standard model is embedded in a supersymmetric grand unified theory (GUT). But it is only possible to accept the possibility of grand unified theories if an acceptable reason for the large separation in mass scales between the Higgs doublet and triplet fields is identified. Most models which exist require either fine tuning or have a very complicated field content and/or do not take into account the possible nonrenormalizable operators, suppressed by the Planck mass.

In order to avoid fine tuning, several solutions to the doublet-triplet splitting problem have been proposed. They include the sliding singlet [回], the missing partner mechanism [2, 3], and an $\mathrm{SO}(10)$ based solution [5, 6]. The sliding singlet solution has been shown to be unstable to radiative corrections [4], and is therefore unacceptable. The missing partner mechanism will in general have problems when nonrenormalizable operators are included in the Hamiltonian, which will generate a mass for the doublet Higgs. The SO(10) models might work, but they often require a large field content [6] so that asymptotic freedom of the GUT-theory is destroyed; thus nonrenormalizable operators are suppressed only by the scale where the GUT-coupling becomes strong (this scale can be significantly lower than the Planck-scale).

One of the most economical and satisfying explanations for why the Higgs doublets are light could be that they are pseudo-Goldstone bosons (PGB's) of a spontaneously broken accidental global symmetry of the Higgs sector [7]. The Higgs sector of the chiral superfields is defined with the use of matter parity. Under this $Z_{2}$ symmetry all matter fields (fermion fields) change sign while the Higgs fields are invariant. When Yukawa couplings are incorporated (couplings of the Higgs sector to matter fields), the accidental global symmetry is explicitly broken; however, because of supersymmetric nonrenormalization theorems the Higgs masses can only be of order of the supersymmetry breaking, or weak scale.

The first attempts to build such a model were made by requiring that the chiral superfields of a given gauge group are put together into a representation of a bigger global symmetry group [7, 8, 9]. For example the 24,5, $\overline{5}$ and 1 of an $\mathrm{SU}(5)$ gauge group could form the 35 adjoint of $\mathrm{SU}(6)$. While the global $\mathrm{SU}(6)$ breaks to $\mathrm{SU}(4) \otimes \mathrm{SU}(2) \otimes \mathrm{U}(1)$, the gauged $\mathrm{SU}(5)$ breaks to $\mathrm{SU}(3) \otimes \mathrm{SU}(2) \otimes \mathrm{U}(1)$, and the uneaten PGB's are in two $\mathrm{SU}(2)$ doublets $\llbracket 7$, \&. 
Other similar models were discussed in ref. [9].

Unfortunately this model requires even more fine tunings of the parameters of the superpotential than the usual fine tuning solution of the doublet-triplet splitting problem. For example in the $\mathrm{SU}(5)$ model mentioned above this would mean that for the general superpotential

$$
\begin{aligned}
& W=\frac{1}{2} M \operatorname{Tr} \Sigma^{2}+\frac{1}{3} \lambda \operatorname{Tr} \Sigma^{3}+\mu \bar{H} \Sigma H+\alpha \bar{H} H+\rho_{1} Y+\frac{\rho_{2}}{2} Y^{2}+ \\
& \rho_{3} Y^{3}+\rho_{4} \operatorname{Tr} \Sigma^{2} Y+\rho_{5} \bar{H} H Y,
\end{aligned}
$$

where the fields $\Sigma, H, \bar{H}, Y$ are the $\mathrm{SU}(5)$ fields transforming according to $24,5, \overline{5}, 1$ the following relations have to hold in order to have the larger global SU(6) invariance:

$$
\begin{aligned}
& \alpha=M=\rho_{2}, \quad \mu=\lambda, \quad \rho_{3}=-\frac{2}{3}\left(\frac{2}{15}\right)^{\frac{1}{2}} \lambda, \\
& \rho_{4}=\frac{1}{\sqrt{30}} \lambda, \quad \rho_{5}=-2\left(\frac{2}{15}\right)^{\frac{1}{2}} \lambda .
\end{aligned}
$$

These relations are very unlikely to be a result of a symmetry of a higher energy theory. Thus this version does not tell much more than the original fine tuned SU(5) theory.

A much more appealing scenario is that the accidental symmetry of the superpotential arises because two sectors of the chiral superfields responsible for gauge symmetry breaking do not mix and thus the global symmetry of this sector is $G \otimes G$ instead of the original gauge group $G$ [11, 12, 13, 14. This accidental symmetry could be a result of a discrete symmetry that forbids the mixing of the two sectors so this scenario might well be a consequence of a symmetry of a larger theory. During spontaneous symmetry breaking $G \otimes G \rightarrow G_{1} \otimes G_{2}$ while the diagonal $G$ (which is the original gauge group) breaks to $\mathrm{SU}(3) \otimes \mathrm{SU}(2) \otimes \mathrm{U}(1)$.

The D-terms of the group $G \otimes G$ in this scheme of spontaneous symmetry breaking (SSB) vanish in order to preserve supersymmetry. Because supersymmetry is preserved, the requirement for "total doubling" [7] is fulfilled, so associated to every Goldstone boson there is also a pseudo-Goldstone boson in a chiral multiplet which is massless only by supersymmetry. Therefore, all the scalars in a Goldstone chiral superfield are light, not only one of the scalar components. We will refer to both as PGB's throughout the paper. The genuine Goldstone bosons remain massless even after adding the soft SUSY breaking terms, while the pseudo-Goldstone bosons get masses at the order of the weak scale at this stage. The 
remaining massless states get masses during the running down from the GUT scale to the weak scale due to the symmetry breaking Yukawa couplings.

These Yukawa couplings have to break the accidental global symmetry of the Higgs sector explicitly. Otherwise the couplings of the Higgs doublets (which are identified with the uneaten PGB's of the broken global symmetry) to the light fermions would vanish. Thus there would be no source for the light fermion masses. The nonvanishing of the couplings of the Higgs fields to the light fermions (especially to the top quark) is also essential for radiative electroweak breaking. Thus it is necessary that in these models the Yukawa couplings explicitly break the accidental symmetry of the Higgs sector.

Explicit symmetry breaking terms in the Higgs sector can yield additional contributions to the $\mu$-term of the Higgs potential (the models presented in section 4 will contain such explicit breaking terms). There can also be additional contributions to the $\mu$-term from nonrenormalizable contributions to the Kähler potential [10].

An example of models of this kind was given in refs. [11, 12, 13, 14]. In this case $G=$ $\mathrm{SU}(6)$, and the accidental global symmetry is broken to $\mathrm{SU}(4) \otimes \mathrm{SU}(2) \otimes \mathrm{U}(1) \otimes \mathrm{SU}(5)$. There are exactly two light doublets in this model, so the low energy particle content is just that of the MSSM. The Higgses are naturally light (they are PGB's), while the triplets have masses of $\mathcal{O}\left(M_{G U T}\right)$.

Although such a model is very appealing in principle, it is not clear that it holds up to more detailed scrutiny. The first problem is to construct a potential with the desired symmetries and symmetry breakings. The second problem is to generate a fermion mass spectrum compatible with observation.

The flavor problem can be addressed by enhancing the field content and including nonrenormalizable operators [13, 14]. However, the first problem is very difficult. The models of refs. [12, 13] do not give the correct minimum without fine tuning and are therefore unacceptable. The model of ref. [14 has the correct symmetry and gives the desired minimum if one incorporates only the renormalizable terms in the superpotential. However, once nonrenormalizable terms are incorporated it is very difficult to construct acceptable models without fine tuning.

In this paper we outline many constraints for model building. We consider only models of the second type; that is, models where the accidental global symmetry arises as a result of two nonmixing sectors of the Higgs fields. In supersymmetric theories it may happen that some 
operators are unexpectedly missing from the superpotential even though they are allowed by all symmetries of the theory. However we will use the most pessimistic assumption, that is, all terms consistent with all the symmetries are present and they are as big as they can be (suppressed only by the appropriate powers of $M_{P l}$ ). Our philosophy for constructing models is to find discrete symmetries that forbid the dangerous mixings of the two sectors. These could be either R-type or usual discrete symmetries. (We assume that all other symmetries of the theory above the Planck scale are broken with the exception of some possible discrete symmetries. These should actually be gauge type discrete symmetries so that they are not destroyed by large gravitational corrections. This implies that these discrete symmetries could possibly have anomalies. However these discrete anomalies can always be canceled by adding extra gauge singlets transforming nontrivially under the discrete symmetries.)

We find for such models a general feature that the more one suppresses mixing terms, the more fine tuning is necessary in the superpotential to maintain the correct values of the vacuum expectation values (VEV's) if the VEV's of all fields in the Higgs sector are comparable. To find a way out we either need to introduce small mass parameters or fields with small (or zero) VEV's.

However, the small parameter which can be used to build these models is necessarily present in models with supersymmetry breaking. Based on the analysis of requirements for a successful model, we show how to exploit the supersymmetry breaking scale to create models which have an accidental global $\mathrm{SU}(6) \otimes \mathrm{SU}(6)$ symmetry. In these models the Higgs is naturally light, and there are no problems with the triplets. Alternatively we will show how to use the second possibility (the presence of fields with zero VEV's) to build another class of natural models without the use of any small parameter.

One can ask whether there are perhaps other models that use an alternative gauge group for which it is simpler to construct an acceptable potential. We will demonstrate that all models that use only regular group embeddings and have no extra light triplet fields are trivial generalizations of the SU(6) model, and are therefore no better (and probably worse).

The outline of this paper is as follows. In the following section, we review the $\mathrm{SU}(6)$ model and give a simple example for a model that is acceptable if one incorporates only renormalizable terms into the superpotential. In section 3 we first discuss the requirements for building an acceptable potential and show why it is difficult to get a natural model. In the first subsection we consider the use of alternative $\mathrm{SU}(6)$ representations to forbid the 
dangerous mixing terms while in the second we discuss the possibility of using restrictive discrete symmetries for this purpose. We draw the conclusion that if mixing terms are suppressed there must be either small parameters or fields with zero VEV in the theory. In section 4 we present three different models that naturally fulfill all the requirements for the superpotential.

The first model uses a small mass parameter, namely the weak scale, to get the correct magnitudes of VEV's of the fields in the Higgs sector. The second model does not use any small mass parameter, but exploits the presence of fields with zero VEV's to obtain an acceptable theory. In the third model, we assume the appearance of the GUT scale by an unspecified dynamical origin. With these three models we demonstrate that the idea of having the Higgses as pseudo-Goldstone particles can be naturally implemented. In section 5 , we consider the possibilities for generalizing the $\mathrm{SU}(6)$ model to $\mathrm{SU}(\mathrm{n})$ groups. Orthogonal groups and the exceptional group $\mathrm{E}_{6}$ are considered in appendix $\mathrm{A}$. We conclude in section 6. Appendix B treats the question of generating the fermion masses.

\section{A Review of the SU(6) Model}

In the SU(6) model of refs. [11, 12, 13, 14, the gauge group of the high energy GUT theory is $\mathrm{SU}(6)$. The accidental symmetry of the Higgs part of the superpotential arises because there are two sectors involving two different fields which do not mix in the potential, so that an accidental global $\mathrm{SU}(6) \otimes \mathrm{SU}(6)$ symmetry is preserved. The fields suggested in refs. 11, 12, 13 to realize this idea were $\Sigma$ in an adjoint 35 representation and $H, \bar{H}$ in $6, \overline{6}$ representations of $\mathrm{SU}(6)$. Their $\mathrm{SU}(5)$ decomposition is

$$
\begin{aligned}
\Sigma & =35=24+6+\overline{6}+1 \\
H & =6=5+1 \\
\bar{H} & =\overline{6}=\overline{5}+1 .
\end{aligned}
$$

Then one of the sectors consists of the fields $H, \bar{H}$ and the other of $\Sigma$. The accidental symmetry is realized if mixing terms of the form $\bar{H} \Sigma H$ are not present in the superpotential. 
If the fields $\Sigma$ and $H, \bar{H}$ develop VEV's of the form

$$
\langle\Sigma\rangle=V\left(\begin{array}{cccccc}
1 & & & & & \\
& 1 & & & & \\
& & 1 & & & \\
& & & 1 & & \\
& & & -2 & \\
& & & & -2
\end{array}\right), \quad\langle H\rangle=\langle\bar{H}\rangle=U\left(\begin{array}{l}
1 \\
0 \\
0 \\
0 \\
0 \\
0
\end{array}\right)
$$

then one of the global $\mathrm{SU}(6)$ factors breaks to $\mathrm{SU}(4) \otimes \mathrm{SU}(2) \otimes \mathrm{U}(1)$, while the other to $\mathrm{SU}(5)$. Together, the VEV's break the gauge group to $\mathrm{SU}(3) \otimes \mathrm{SU}(2) \otimes \mathrm{U}(1)$.

The Goldstone bosons (GB's) coming from the breaking $\mathrm{SU}(6) \rightarrow \mathrm{SU}(4) \otimes \mathrm{SU}(2) \otimes \mathrm{U}(1)$ are (according to their $\mathrm{SU}(3) \otimes \mathrm{SU}(2) \otimes \mathrm{U}(1)$ transformation properties):

$$
(\overline{3}, 2)_{\frac{5}{6}}+(3,2)_{-\frac{5}{6}}+(1,2)_{\frac{1}{2}}+(1,2)_{-\frac{1}{2}}
$$

while from the breaking $\mathrm{SU}(6) \rightarrow \mathrm{SU}(5)$ the GB's are

$$
(3,1)_{-\frac{1}{3}}+(\overline{3}, 1)_{\frac{1}{3}}+(1,2)_{\frac{1}{2}}+(1,2)_{-\frac{1}{2}}+(1,1)_{0}
$$

But the following GB's are eaten by the heavy vector bosons due to the supersymmetric Higgs mechanism (the gauge symmetry is broken from $\mathrm{SU}(6)$ to $\mathrm{SU}(3) \otimes \mathrm{SU}(2) \otimes \mathrm{U}(1)$ ):

$$
(3,1)_{-\frac{1}{3}}+(\overline{3}, 1)_{\frac{1}{3}}+(3,2)_{-\frac{5}{6}}+(\overline{3}, 2)_{\frac{5}{6}}+(1,2)_{\frac{1}{2}}+(1,2)_{-\frac{1}{2}}+(1,1)_{0}
$$

Thus exactly one pair of doublets remains uneaten which can be identified with the Higgs fields of the MSSM. One can show that the uneaten doublets are in the following combinations of the fields $\Sigma, H, \bar{H}$ :

$$
\begin{aligned}
& h_{1}=\frac{U h_{\Sigma}-3 V h_{H}}{\sqrt{9 V^{2}+U^{2}}}, \\
& h_{2}=\frac{U \bar{h}_{\Sigma}-3 V \bar{h}_{\bar{H}}}{\sqrt{9 V^{2}+U^{2}}},
\end{aligned}
$$

where $h_{H}$ and $\bar{h}_{\bar{H}}$ denote the two doublets living in the $\mathrm{SU}(6)$ field $H$ and $\bar{H}$, while $h_{\Sigma}$ and $\bar{h}_{\Sigma}$ denote the two doublets living in the $\mathrm{SU}(6)$ adjoint $\Sigma$.

In order to get the correct order of symmetry breaking we need to have $\langle\Sigma\rangle \sim M_{G U T}$, $\langle H\rangle=\langle\bar{H}\rangle>\langle\Sigma\rangle$. In this case the gauge group is broken as 


$$
\mathrm{SU}(6) \rightarrow \mathrm{SU}(5) \rightarrow \mathrm{SU}(3) \otimes \mathrm{SU}(2) \otimes \mathrm{U}(1)
$$

In the case of opposite ordering of the magnitudes of the VEV's we would get

$$
\mathrm{SU}(6) \rightarrow \mathrm{SU}(4) \otimes \mathrm{SU}(2) \otimes \mathrm{U}(1) \rightarrow \mathrm{SU}(3) \otimes \mathrm{SU}(2) \otimes \mathrm{U}(1)
$$

which would give unreasonably large threshold correction to the RG values of $\sin ^{2} \theta_{W}$.

The biggest question of this model is how to realize the necessary suppression of mixing terms like $\bar{H} \Sigma H$ in the superpotential and thus achieve the desired vacuum. We want to find discrete symmetries that forbid the mixing of the two sectors. These could be either R-type or usual discrete symmetries.

In ref. [12 a $Z_{2}$ discrete symmetry $\bar{H} \rightarrow-\bar{H}, S \rightarrow-S, H \rightarrow H, \Sigma \rightarrow \Sigma(S$ is an $\mathrm{SU}(6)$ singlet) was suggested to forbid the mixing term $\bar{H} \Sigma H$. But in the supersymmetric limit the $H, \bar{H}, S$ VEV's were all zero, so these VEV's come from the soft breaking terms, and consequently

$$
\langle H\rangle \sim\left(m M_{G U T}\right)^{\frac{1}{2}} \approx 10^{8} \mathrm{GeV}
$$

where $m$ is a mass parameter of the order of the weak scale. Consequently unreasonably large fine tuning is needed to obtain $\langle H\rangle>M_{G U T}$.

One can overcome this problem by introducing more fields into the theory [14]. One can take for example two adjoints $\Sigma_{1}, \Sigma_{2}$ instead of just one and a discrete $Z_{3}$ symmetry under which $\Sigma_{1} \rightarrow e^{\frac{2 \pi i}{3}} \Sigma_{1}$ and $\Sigma_{2} \rightarrow e^{-\frac{2 \pi i}{3}} \Sigma_{2}$, while $H, \bar{H}, S$ are invariant. Then the most general renormalizable superpotential is of the form

$$
\begin{aligned}
& W\left(S, H, \bar{H}, \Sigma_{1}, \Sigma_{2}\right)=a S\left(\bar{H} H-\mu^{2}\right)-\frac{M^{\prime}}{2} S^{2}-\frac{\gamma}{3} S^{3}-m \bar{H} H+ \\
& \alpha S \operatorname{Tr} \Sigma_{1} \Sigma_{2}+M \operatorname{Tr} \Sigma_{1} \Sigma_{2}+\frac{\lambda_{1}}{3} \operatorname{Tr} \Sigma_{1}^{3}+\frac{\lambda_{2}}{3} \operatorname{Tr} \Sigma_{2}^{3} .
\end{aligned}
$$

which automatically has the global $\mathrm{SU}(6) \otimes \mathrm{SU}(6)$ symmetry. The VEV's are:

$$
\langle S\rangle=\frac{m}{a}
$$




$$
\begin{aligned}
& \left\langle\Sigma_{1}\right\rangle=\frac{\alpha \frac{m}{a}+M}{\left(\lambda_{1}^{2} \lambda_{2}\right)^{\frac{1}{3}}}\left(\begin{array}{cccccc}
1 & & & & & \\
& 1 & & & & \\
& & 1 & & & \\
& & & 1 & & \\
& & & -2 & \\
& & & & -2
\end{array}\right)
\end{aligned}
$$

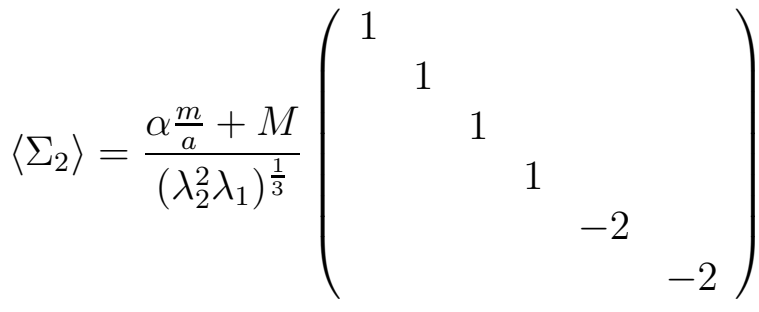

$$
\begin{aligned}
& \langle H\rangle=\langle\bar{H}\rangle=\left[\mu^{2}+\frac{M^{\prime} m}{a^{2}}+\frac{\gamma m^{2}}{a^{3}}-\frac{12\left(\alpha \frac{m}{a}+M\right)^{2} \alpha m}{a^{2} \lambda_{1} \lambda_{2}}\right]^{\frac{1}{2}}\left(\begin{array}{l}
1 \\
0 \\
0 \\
0 \\
0 \\
0
\end{array}\right) \text {. }
\end{aligned}
$$

so that this model gives the correct order of VEV's if $m, M, M^{\prime} \sim M_{G U T}$. There is no renormalizable mixing term allowed by the discrete symmetry that could destroy the accidental $\mathrm{SU}(6) \otimes \mathrm{SU}(6)$ symmetry.

But the problem is that it is not sufficient to consider only renormalizable operators. Nonrenormalizable operators scaled by inverse power of $M_{P l}$ can potentially introduce large breaking of the global $\mathrm{SU}(6) \otimes \mathrm{SU}(6)$ symmetry, which in turn yields large contributions to the PGB masses. In particular, in the above example the term $\frac{1}{M_{P l}} \bar{H} \Sigma_{1} \Sigma_{2} H$ is allowed and gives an unacceptably big correction to the PGB masses if present. Namely, the Higgs doublets would acquire masses $\sim M_{G U T}^{2} / M_{P l} \approx 10^{13} \mathrm{GeV}$.

\section{Requirements and Constraints for the Superpoten- tial}

We have seen in the previous section that even if mixing terms of the renormalizable superpotential are forbidden by some discrete symmetries, the possible nonrenormalizable operators can still break the accidental global symmetry and thus spoil the solution to the doublet triplet splitting problem. 
The origin of the nonrenormalizable operators can be of two forms: they either come from integrating out heavy $\left(\mathcal{O}\left(M_{P l}\right)\right)$ particles from tree level diagrams or they can be a consequence of nonperturbative effects.

The dangerous mixing terms coming from integrating out the heavy fields can be easily forbidden by some additional requirements on the Planck scale particles, for example by requiring that all the Planck mass fields are matter (fermion) fields. In this case the nonrenormalizable terms arising from integrating out the heavy fields can only yield Yukawa terms. But we know that Yukawa terms are irrelevant from the point of view of PGB masses (the accidental global symmetry is a symmetry of the Higgs sector only). This assumption on the heavy fields is usually fulfilled by the interaction terms introduced in models for light fermion masses (e.g. [13, 14]). In those models we want to generate exactly additional Yukawa terms suppressed by Planck masses. Thus matter parity can be used to forbid all dangerous nonrenormalizable mixing terms arising from tree diagrams. Loop diagrams are naturally proportional to supersymmetry breaking.

Even if the above assumption for the superpotential involving heavy fields is valid, there is still the possibility of Planck mass suppressed operators in the superpotential which violate the global symmetry. Although the nonrenormalization theorem prevents these operators from being generated perturbatively if they were not present at tree level, we will take the attitude that all operators consistent with the low energy gauge and discrete symmetries are present, both in the Kähler potential and in the superpotential. We ask the question whether it is possible with this assumption to still maintain an approximate global symmetry which can guarantee that the Higgs doublet is sufficiently light.

The first observation is that the Kähler potential will always permit symmetry breaking terms, suppressed only by two powers of $M_{P l}$, for example:

$$
\frac{1}{M_{P l}^{2}} H^{\dagger} \Sigma^{\dagger} \Sigma H
$$

There is no symmetry which can prevent such a term. However, although such terms do break the accidental global symmetry, they do not lead to generation of a mass term for the PGB's.

However, the PGB mass terms will be generated if the global symmetry is broken in the superpotential. In the remainder of this section, we show that it is extremely difficult to prevent mixing in the superpotential. 
We now summarize the requirements for the superpotential of a realistic model.

1. The mass terms for the PGB's (which are identified with the Higgs doublets of the MSSM) resulting from the symmetry breaking mixing terms should be suppressed at least by a factor of $10^{-13}$ compared to the GUT scale. In this case the masses of the PGB's will be at the order of $1000 \mathrm{GeV}$.

2. The VEV's of the fields $\Sigma$ and $\bar{H}, H$ should be naturally (without tuning) at the order of the GUT scale $\left(10^{16} \mathrm{GeV}\right)$.

3. The triplets contained in the $\Sigma$ field should have GUT-scale masses not to cause too large proton decay. One might think that the same requirement holds for the triplets contained in the fields $\bar{H}, H$. However these triplets are eaten by the heavy $\mathrm{SU}(6)$ gauge bosons and are not dangerous for proton decay.

There are two approaches one could imagine to prevent mixing through nonrenormalizable operators. One might try to find a representation of $\mathrm{SU}(6)$ which breaks $\mathrm{SU}(6)$ to $\mathrm{SU}(4) \otimes \mathrm{SU}(2) \otimes \mathrm{U}(1)$ but does not allow mixing. Alternatively, one can search for more restrictive discrete symmetries.

We have found no solution with alternative representations. It is also very difficult to realize the second solution if we try to use $M_{P l}$ as the only mass scale in the theory. We show that one either needs to introduce small mass scales into the theory or to use fields that have zero VEV's to overcome all constraints listed in the following subsections.

In the next two subsections we consider the above two possibilities for model building. We show that the above requirements necessarily lead us to consider the kind of models presented in section 4 .

\subsection{Alternative representations}

Let us first consider the possibility of achieving the desired symmetry breaking pattern with alternative representations of $\mathrm{SU}(6)$. One can consider symmetric, antisymmetric, or mixed representations. We don't want to replace the the $H, \bar{H}$ fields because the $\bar{H}$ field is capable of splitting the light fermions from the heavy ones through the renormalizable operator $15 \bar{H} \overline{6}$, see [13], so we only consider replacing the $\Sigma$ field. If the representation is symmetric, one does not achieve the desired symmetry breaking pattern. An antisymmetric

representation (for example a 15 looks promising) can achieve a good symmetry breaking pattern since it can break $\mathrm{SU}(6)$ to $\mathrm{SU}(4) \otimes \mathrm{SU}(2)$. Thus with an additional U(1) gauge group 
$\mathrm{SU}(6) \otimes \mathrm{U}(1)$ could break to $\mathrm{SU}(4) \otimes \mathrm{SU}(2) \otimes \mathrm{U}(1)$ (much like the flipped $\mathrm{SU}(5)$ model of [3]). Furthermore, it looks naively as if it can forbid undesired mixing terms such as $15 \overline{6} \overline{6}$ because of the antisymmetry of $15_{i j}$. However, in order to cancel anomalies, one must introduce additional fields, either $\overline{15}$ or $\overline{6}+\overline{6}^{\prime}$. But this addition makes mixing already possible through $15 \overline{15} \bar{H} H$ or in the other case through $15 \bar{H} \overline{6}$. Larger representations do not help because we require a representation that is capable to break $\mathrm{SU}(6)$ to $\mathrm{SU}(4) \otimes \mathrm{SU}(2) \otimes \mathrm{U}(1)$.

\subsection{Discrete symmetries}

The next possibility is to look for more restrictive discrete symmetries. Throughout this subsection we will assume that the only mass scale present in the theory is $M_{P l}$ and that all fields have VEV's of the order of the GUT scale. It turns out that under these assumptions even with additional fields, it is extremely difficult to find a satisfactory superpotential with no unnaturally small parameter. We first summarize the reasons why it is difficult to find a satisfactory potential without fine tuning. We subsequently elaborate and illustrate each point in more detail. To be explicit, we assume all fields in the Higgs sector have VEV's of order $10^{-3} M_{P l}$, the lowest possible value, in order to obtain the maximum suppression in higher dimension mixing operators. This ratio might in fact be larger; one would then need to suppress mixing operators still further. For this value, we require that the mixing term is at least of dimension four greater than the terms in the superpotential which respect the symmetry and generate the VEV's for the $\Sigma$ and $H, \bar{H}$ fields. The PGB masses will then be at most $\left(M_{G U T} / M_{P l}\right)^{4} M_{G U T} \sim 1000 \mathrm{GeV}$.

It is easy to see that with just the fields $\Sigma, H, \bar{H}$ we can not obtain a successful superpotential. The reason for this is that in order to get nonzero VEV's for the fields we need to have at least two terms in both sectors of the accidental global symmetry (one sector contains the adjoint $\Sigma$ and breaks $\mathrm{SU}(6)$ to $\mathrm{SU}(4) \otimes \mathrm{SU}(2) \otimes \mathrm{U}(1)$ while the other $H$ and $\bar{H}$ and breaks $\mathrm{SU}(6)$ to $\mathrm{SU}(5))$. Then the quotient of the two terms can always multiply a term in the other sector, thereby generating unwanted mixing. Explicitly, if there are terms in one sector of the form

$$
\frac{1}{M_{P l}^{a-3}} \operatorname{Tr} \Sigma^{a}+\frac{1}{M_{P l}^{b-3}} \operatorname{Tr} \Sigma^{b},
$$

then $\operatorname{Tr} \Sigma^{b-a}$ transforms trivially under an abelian discrete symmetry (even if it is an R-type 
symmetry). The presence of the terms

$$
\frac{1}{M_{P l}^{2 c-3}}(\bar{H} H)^{c}+\frac{1}{M_{P l}^{2 d-3}}(\bar{H} H)^{d}
$$

in the other sector then means that terms such as

$$
\frac{1}{M_{P l}^{2 c+b-a-3}}\left(\bar{H} \Sigma^{b-a} H\right)(\bar{H} H)^{c-1}
$$

are allowed. The number $b-a$ cannot be arbitrarily big if the dimensionful fields have VEV's of order $M_{G U T}$. This is because in order to balance the two terms in equation 3.15, there must be a small coefficient of order $\epsilon_{G}^{b-a}$ where $\epsilon_{G}=M_{G U T} / M_{P l} \approx 10^{-3}$. So in order for the mixing term to be suppressed by $10^{-13}$, the mixing must be suppressed by at least $\epsilon_{G}^{4}$. But then $b-a \geq 4$ and there must be a small parameter in the potential of order $\epsilon_{G}^{4}$, which is badly fine tuned.

So we have established that one requires additional fields, that there must be at least two operators in each of the two nonmixing sectors (one involving the $H$ and $\bar{H}$ fields and one involving only the $\Sigma$ field), and that the quotient of operators in the superpotential from the same sector must involve negative powers of at least one field, so that such symmetry invariants are not holomorphic functions of the fields.

The next point is that in order to prevent fine tuning, the superpotential should contain operators of similar dimension. The argument which we just gave without additional singlets can readily be generalized (if the singlet VEV is of the same order as those of other fields) to show that in order to prevent fine tuning, the dimension of the operators in the potential which are balanced at the minimum should have comparable dimension. Furthermore, a term of dimension $d$ will yield a mass term for the non PGB's of the order $M \approx M_{G U T} \epsilon_{G}^{d-3}$. A very high dimension operator without a large coefficient will yield masses for the triplet fields much less than $M_{G U T}$. Thus according to our requirement 3 the terms containing the $\Sigma$ field should have low dimensions so that the triplets contained in $\Sigma$ have sufficiently large masses.

Of course, one can consider cases where not all VEV's are the same, but then VEV's are larger than $M_{G U T}$ and mixing terms will be less suppressed.

We can generalize the above argument about the superpotential containing only the fields $\Sigma, \bar{H}, H$ to the case when the superpotential also includes an additional SU(6) singlet. To 
have nonzero VEV's for the fields we need at least two terms that contain $\bar{H} H$ and two that contain $\Sigma$ in the superpotential, while all these four terms may contain the SU(6) singlet field $S$. Thus generally the superpotential will have the form (if there is no mixing of the two sectors)

$$
(\bar{H} H)^{a} S^{b}+(\bar{H} H)^{c} S^{d}+\operatorname{Tr} \Sigma^{e} S^{f}+\operatorname{Tr} \Sigma^{g} S^{h} .
$$

Without loss of generality we can assume that $d>b, f>h$ and $d>f$. If $f>h$ we require that $g>e$; otherwise the operator $\operatorname{Tr} \Sigma^{e-g} S^{f-h}$ (which is just the quotient of the last two terms and thus invariant under all discrete symmetries) would be holomorphic and could multiply either term of the $\Sigma$ sector to give a nonsuppressed mixing term. But because $g>e$, the operator $\Sigma^{g-e} S^{h-f+d}(\bar{H} H)^{c}$ is holomorphic. This operator is allowed by the discrete symmetries, because it is the product of two terms of the superpotential divided by a third term. Therefore the dimension of the allowed mixing term is equal to the dimension of one of the terms originally present in the superpotential plus the difference of the dimension of two terms present in the superpotential. (It is easy to see that this is also true for the case $e>g$.) Thus the necessary fine tuning is equal to the suppression factor of the mixing term. If we want to suppress mixing by $\epsilon_{G}^{4}$ we will need fine tuning of the same order (to balance terms of different dimensions). To illustrate this argument we present a model where although mixing terms are suppressed sufficiently we need unreasonably large fine tuning to get the correct VEV's. In this model the superpotential is given by

$$
\begin{aligned}
& W(\Sigma, H, \bar{H}, S)=\frac{\alpha}{M_{P l}^{4}} S^{5} \bar{H} H+\beta S \operatorname{Tr} \Sigma^{2}+ \\
& \frac{\gamma}{M_{P l}^{3}}(\bar{H} H)^{3}+\frac{\delta}{M_{P l}^{4}} \operatorname{Tr} \Sigma^{7},
\end{aligned}
$$

where the discrete charges for the fields $\Sigma, \bar{H} H, S$ are $Q_{\Sigma}=\frac{25}{61}, Q_{\bar{H} H}=\frac{38}{61}, Q_{S}=\frac{3}{61}$ and the R-charge of the superpotential is $\frac{53}{61}$. (The transformation of the fields under the discrete symmetry is given by $\Phi \rightarrow e^{2 \pi i Q} \Phi$.) Then the first allowed mixing term is $\bar{H} \Sigma^{5} H S^{4}$, suppressed by 4 dimensions compared to $\operatorname{Tr} \Sigma^{7}$ or $S^{5} \bar{H} H$. The equations of motion for this theory are

$$
\begin{aligned}
& \frac{5 \alpha S^{4}(\bar{H} H)}{M_{P l}^{4}}+\beta \operatorname{Tr} \Sigma^{2}=0 \\
& \frac{\alpha S^{5}}{M_{P l}}+3 \gamma(\bar{H} H)^{2}=0
\end{aligned}
$$




$$
2 \beta S \Sigma+\frac{7 \delta}{M_{P l}^{4}}\left(\Sigma^{6}-\frac{1}{6} \operatorname{Tr} \Sigma^{6}\right)=0 .
$$

If the VEV of $\Sigma$ has the form

$$
\langle\Sigma\rangle=V\left(\begin{array}{llllll}
1 & & & & & \\
& 1 & & & & \\
& & 1 & & & \\
& & & 1 & & \\
& & & & -2 & \\
& & & & & -2
\end{array}\right)
$$

then the solution for $V$ is

$$
V=M_{P l}\left[\left(\frac{-3 \gamma}{\alpha}\right)\left(\frac{12 \beta}{5 \alpha}\right)^{2}\left(\frac{2 \beta}{147 \delta}\right)^{13}\right]^{\frac{1}{61}} .
$$

The number multiplying $M_{P l}$ should be $10^{-3}$, so even if we assume that this is the $13 / 61$ st power of a combination of the parameters this combination must be $\left(10^{-3}\right)^{\frac{61}{13}} \sim 10^{-12}$. Thus we can see explicitly in this model that the amount of fine tuning $\left(10^{-12}\right)$ is equal to the suppression factor of the dangerous mixing terms.

One might think that we can overcome this problem by introducing even more fields into the theory. If we could find a superpotential where the number of terms contained in the superpotential is equal to the number of fields in the superpotential we could assign arbitrarily different R-charges to the fields in the superpotential and thus forbid mixing terms. However this is not possible. The reason is the following: suppose we have $\mathrm{n}$ fields and $\mathrm{n}$ polynomial terms in the superpotential. Let's call these terms $A_{i}, i=1, \ldots \mathrm{n}$, where $A_{i}$ is a polynomial of the fields $\Phi_{k}, k=1, \ldots \mathrm{n}$. The superpotential is then

$$
W\left(\Phi_{i}\right)=\sum_{k=1}^{n} \alpha_{k} A_{k}\left(\Phi_{i}\right)
$$

The equations of motion are $\frac{\partial W}{\partial \Phi_{i}}=0$. If neither of the VEV's is zero then we can also write these equations in the form $\Phi_{i} \frac{\partial W}{\partial \Phi_{i}}=0, i=1, \ldots \mathrm{n}$. Thus we get a system of equations

$$
\sum_{k=1}^{n} \beta_{i k} \bar{A}_{k}=0, \quad i=1, \ldots \mathrm{n},
$$

where $\bar{A}_{k}=A_{k}\left(\left\langle\Phi_{j}\right\rangle\right)$. This is a set of $\mathrm{n}$ linear homogeneous equations for the terms $\bar{A}_{k}$. There are two possibilities: the determinant of the coefficients $\beta_{i k}$ is either zero or nonzero. 
To have it zero requires fine tuning of the parameters in the superpotential and even then we can not have all VEV's determined by the superpotential because the equations are linearly dependent so there are in fact fewer equations than $\mathrm{n}$. If the determinant is nonzero then the only possibility is to have $\bar{A}_{k}=0$ for $k=1, \ldots \mathrm{n}$. This implies that at least one of the VEV's is zero contrary to our assumption.

Thus we need at least $n+1$ terms in the superpotential to have the VEV's of all fields determined of the correct size without fine tuning. But this means that we can not choose the R-charges of the fields arbitrarily. Generally these connections among the R-charges make it very difficult to find an acceptable superpotential that both determines the VEV's at the right scale without fine tuning and has the mixing terms sufficiently suppressed. In all cases we examined with only low dimensional operators for the $\Sigma$ field in the superpotential we were either able to find allowed unsuppressed mixing terms or fine tuning was required to set the VEV's to the right scale.

\section{Three Models in which the Supersymmetric Higgs Particles are Naturally Pseudo-Goldstone Bosons}

We have shown in the preceding section that one cannot construct a model based on low energy discrete symmetries without a small parameter if neither of the VEV's of the fields of the Higgs sector is zero. However, low energy supersymmetry must contain a small parameter, namely the weak scale, or equivalently, the supersymmetry breaking scale. In the first subsection, we show how one can exploit this small parameter to generate models which naturally respect the accidental global $\mathrm{SU}(6) \otimes \mathrm{SU}(6)$ symmetry. Our models differ from the model in ref. [12] in that we exploit the supersymmetry breaking scale, but we do not need to tune the parameters. We naturally balance small terms against each other.

In the second subsection we present a different class of models. These models contain fields with zero VEV's; thus the no-go arguments of the previous section are not valid here. These models include two mass parameters: all mass terms are proportional to the GUTscale while the nonrenormalizable operators are suppressed by the Planck-scale. In the third model we assume the appearance of a dynamical scale (related to the GUT scale) but do not specify its origin.

These three models serve as existence proofs for models which implement the $\mathrm{SU}(6) \otimes$ 
$\mathrm{SU}(6)$ symmetry. Based on the considerations of the previous section, we expect the simplest successful models will have features of one of the models presented below.

\section{$4.1 \quad$ Model 1}

In these models we give superpotentials which together with the soft breaking terms give the correct values of VEV's. This is similar to the model of ref. [12 but there the superpotential contained only renormalizable terms. Consequently the soft breaking terms alone were not enough to set the VEV's to the right scale and additional fine tuning was required.

The essential observation is that the triplets from $H, \bar{H}$ are eaten by the heavy gauge bosons and thus we don't need $\mathcal{O}\left(M_{G U T}\right)$ mass terms for these fields. The superpotential is given by

$$
W(\Sigma, \bar{H}, H)=\frac{1}{2} M \operatorname{Tr} \Sigma^{2}+\frac{1}{3} \lambda \operatorname{Tr} \Sigma^{3}+\alpha \frac{(\bar{H} H)^{n}}{M_{P l}^{2 n-3}}
$$

If one assigns a discrete $Z_{n}$ symmetry under which $\bar{H} H \rightarrow e^{2 \pi i / n} \bar{H} H$ and $\Sigma$ is invariant then these terms are the lowest order allowed ones. In the supersymmetric limit

$$
\langle\Sigma\rangle=\frac{M}{\lambda}\left(\begin{array}{cccccc}
1 & & & & & \\
& 1 & & & & \\
& & 1 & & & \\
& & & 1 & & \\
& & & & -2 & \\
& & & & & -2
\end{array}\right), \quad\langle H\rangle=\langle\bar{H}\rangle=0
$$

The scalar potential (including the soft breaking terms) will have the form:

$$
\begin{aligned}
& V(\Sigma, \bar{H}, H)=\operatorname{Tr}\left|M \Sigma+\lambda \Sigma^{2}-\frac{1}{6} \lambda \operatorname{Tr} \Sigma^{2}\right|^{2}+\frac{n^{2} \alpha^{2}}{M_{P l}^{4 n-6}}(\bar{H} H)^{2 n-2}\left(|H|^{2}+|\bar{H}|^{2}\right)+ \\
& A m \lambda \operatorname{Tr} \Sigma^{3}+A^{\prime} m \alpha \frac{(\bar{H} H)^{n}}{M_{P l}^{2 n-3}}+B M m \Sigma^{2}+m^{2}\left(\operatorname{Tr} \Sigma^{2}+|H|^{2}+|\bar{H}|^{2}\right)+\mathrm{D}-\text { terms }
\end{aligned}
$$

where $m$ is a mass parameter of the order of the weak scale while $A, A^{\prime}, B$ are dimensionless parameters. The D-terms have to vanish not to have supersymmetry breaking in the visible sector. The soft breaking terms shift the $\Sigma$ VEV only by a small $(\sim m)$ amount. However for the $\bar{H}, H$ terms we have the possibility of a new minimum appearing due to the soft 
breaking terms. To find this we minimize the $\bar{H}, H$ part of the potential (using $\langle H\rangle=$ $\langle\bar{H}\rangle=U(1,0,0,0,0,0)$ which is a consequence of the vanishing of the D-terms).

$$
V(U)=\frac{2 n^{2}|\alpha|^{2}}{M_{P l}^{4 n-6}} U^{4 n-2}+\frac{A^{\prime} m \alpha}{M_{P l}^{2 n-3}} U^{2 n}+2 m^{2} U^{2} .
$$

Minimizing this potential we will get for one of the minima

$$
U=\left[\frac{1}{2 n^{2} \alpha(4 n-2)}\left(-n A^{\prime}+\sqrt{n^{2}\left(A^{\prime}\right)^{2}-16 n^{2}(2 n-1)}\right) M_{P l}^{2 n-3} \mu\right]^{\frac{1}{2 n-2}}
$$

The magnitude of the VEV is determined by the factor

$$
\left[M_{P l}^{2 n-3} \mu\right]^{\frac{1}{2 n-2}}=\left(\frac{\mu}{M_{P l}}\right)^{\frac{1}{2 n-2}} M_{P l}
$$

For $n<4$ we get a smaller scale than $M_{G U T}$ which is not acceptable. However for $n \geq 4$ the resulting scale always lies between the GUT scale and the Planck scale. (For $n=4,5,6$ we get $U \approx 1.510^{16}, 710^{16}, 210^{17} \mathrm{GeV}$.) Thus all these cases yield naturally the correct values of the $H, \bar{H}$ VEV's. The first mixing term allowed by the $Z_{n}$ symmetry is $\frac{1}{M_{P l}^{2 n-2}}(\bar{H} H)^{n-1}(\bar{H} \Sigma H)$. However the resulting mass for the PGB's is

$$
U\left(\frac{U}{M_{P l}}\right)^{2 n-3}=\mu\left(\frac{\mu}{M_{P l}}\right)^{2 n-2}<\mu
$$

This means that all models with $n \geq 4$ yield an acceptable theory with the correct order of VEV's and naturally suppressed mixing terms. The possibility that the $H, \bar{H}$ VEV's are between the GUT and the Planck scale may even be welcome from the point of view of fermion masses (see ref. [13]), and $\langle H\rangle>M_{G U T}$ is also required for the unification of couplings.

We can not use this method for getting GUT-scale VEV's for the sector containing the field $\Sigma$ because then the triplets of $\Sigma$ would get too small masses and would spoil the proton stability. This is however not the case for the $\bar{H}, H$ fields because the triplets from $\bar{H}, H$ are eaten by the SU(6) gauge bosons. Fortunately it suffices to use this method for only one of the sectors because then mixing terms are already sufficiently suppressed. This leads us to the choice of the operator $(\bar{H} H)^{n} / M_{P l}^{2 n-3}$, while we have no restriction for the other sector. Alternatively we could use for example the superpotential 


\begin{tabular}{|c|c|c|c|c|c|c|c|c|}
\hline & $\Sigma_{1}$ & $\Sigma_{2}$ & $A$ & $B$ & $T$ & $S$ & $N$ & $H H$ \\
\hline$Z_{3}^{(1)}$ & $\frac{1}{3}$ & $-\frac{1}{3}$ & $-\frac{1}{3}$ & $\frac{1}{3}$ & 0 & 0 & 0 & 0 \\
\hline$Z_{3}^{(2)}$ & 0 & 0 & 0 & 0 & $\frac{1}{3}$ & $-\frac{1}{3}$ & 0 & $\frac{1}{6}$ \\
\hline$Z_{2}$ & 0 & 0 & 0 & 0 & 0 & 0 & 0 & $\frac{1}{2}$ \\
\hline$R$ & $\frac{1}{9}$ & $\frac{2}{9}$ & $-\frac{1}{9}$ & $\frac{4}{9}$ & $\frac{5}{9}$ & $\frac{1}{9}$ & $\frac{2}{3}$ & $\frac{1}{9}$ \\
\hline
\end{tabular}

Table 1: The discrete charge assignments of the fields of the Higgs sector of model 2.

$$
M \operatorname{Tr} \Sigma_{1} \Sigma_{2}+\frac{1}{3} \lambda_{1} \operatorname{Tr} \Sigma_{1}^{3}+\frac{1}{3} \lambda_{2} \operatorname{Tr} \Sigma_{2}^{3}+\frac{\alpha}{M_{P l}^{2 n-3}}(\bar{H} H)^{n}
$$

with the additional $Z_{3}$ discrete charges $Q_{\Sigma_{1}}=1 / 3, Q_{\Sigma_{2}}=-1 / 3, Q_{\bar{H} H}=0$ (similar to the model of ref. [14). In this case the mixing term is even more suppressed by the additional discrete symmetry. The lowest order mixing term in this case is

$$
\left(\bar{H} \Sigma_{1} \Sigma_{2} H\right)(\bar{H} H)^{n-1} / M_{P l}^{2 n-1} .
$$

This model will be used in Appendix B, when we extend it to incorporate fermion masses.

\subsection{Model 2}

In this class of models we will use low dimension operators to get the VEV's of the adjoint sector and then use two singlets with zero VEV's to communicate the required values of the VEV's to the $H, \bar{H}$ fields. The adjoint sector consists of two adjoint fields $\Sigma_{1}, \Sigma_{2}$ and two $\mathrm{SU}(6)$ singlets $A, B$, while we introduce additional singlets $(N, T, S)$ to get the desired VEV's for $H, \bar{H}$. We use a $Z_{3}^{(1)} \otimes Z_{3}^{(2)} \otimes Z_{2} \otimes R$ symmetry, where $R$ is a discrete R-symmetry

with the charge of the superpotential being $\frac{1}{3}$. The discrete charge assignments of the fields are given in table 1.

The lowest order superpotential allowed by the discrete and gauge symmetries is

$$
\begin{aligned}
& M \operatorname{Tr} \Sigma_{1} \Sigma_{2}+a \operatorname{Tr} \Sigma_{1}^{3}+b \operatorname{Tr} \Sigma_{2}^{2} A+M^{\prime} A B+c B^{3}+ \\
& \frac{\alpha}{M_{P l}} N\left(\operatorname{Tr} \Sigma_{2}^{3}+\beta T^{3}\right)+\frac{\gamma}{M_{P l}^{2}} S\left(T^{4}-\delta(\bar{H} H)^{2}\right) .
\end{aligned}
$$

The VEV's are

$$
V_{1}=\left(\frac{M^{6} M^{\prime 3}}{3^{8} 2^{5} a^{5} b^{3} c}\right)^{\frac{1}{9}} \approx\left[M^{6} M^{\prime 3}\right]^{\frac{1}{9}}
$$




$$
\begin{aligned}
& V_{2}=\frac{3 a}{M} V_{1}^{2} \approx\left[M^{3} M^{\prime 6}\right]^{\frac{1}{9}} \\
& \langle B\rangle=-\frac{108 b a^{2}}{M^{2} M^{\prime}} V_{1}^{4} \approx\left[M^{6} M^{\prime 3}\right]^{\frac{1}{9}} \\
& \langle A\rangle=-\frac{3 c}{M^{\prime}}\langle B\rangle^{2} \approx\left[M^{3} M^{\prime 6}\right]^{\frac{1}{9}} \\
& \langle T\rangle=\left(\frac{12}{\beta}\right)^{\frac{1}{3}} V_{2} \approx\left[M^{3} M^{\prime 6}\right]^{\frac{1}{9}} \\
& \langle H\rangle=\langle\bar{H}\rangle=\frac{\langle T\rangle}{\delta^{\frac{1}{4}}} \approx\left[M^{3} M^{\prime 6}\right]^{\frac{1}{9}} \\
& \langle S\rangle=\langle N\rangle=0
\end{aligned}
$$

where $V_{1}$ and $V_{2}$ are defined by

$$
\left\langle\Sigma_{1}\right\rangle=V_{1}\left(\begin{array}{cccccc}
1 & & & & & \\
& 1 & & & & \\
& & 1 & & & \\
& & 1 & & \\
& & & -2 & \\
& & & & -2
\end{array}\right), \quad\left\langle\Sigma_{2}\right\rangle=V_{2}\left(\begin{array}{cccccc}
1 & & & & & \\
& 1 & & & & \\
& & 1 & & & \\
& & & 1 & & \\
& & & -2 & \\
& & & & & -2
\end{array}\right)
$$

If $M, M^{\prime} \approx M_{G U T}$ then all fields (with the exception of $N$ and $S$ ) have $\mathcal{O}\left(M_{G U T}\right)$ VEV's. The lowest possible mixing term in the superpotential is $\left(\bar{H} \Sigma_{1} H\right)(\bar{H} H) A S$ which yields a supersymmetric mass term (so called $\mu$-term) for PGB Higgs doublets $\mu \sim\left(10^{16} / 210^{19}\right)^{4} 10^{16} \approx$ $1000 \mathrm{GeV}$. One can see that the dangerous mixing term is quite big (compared to the lowest order mixing term of Model 1). One might need some additional suppression factor but no large fine tuning. The feature of this model that there are symmetry breaking terms that yield extra $\mu$-terms for the Higgs doublets (which may also arise in the models presented in the previous subsection) solve a potential problem of these models. Namely, if there are no explicit symmetry breaking terms in the Higgs sector then the 'genuine GB's' will remain exactly massless at the GUT scale even after adding the soft breaking terms. This results in a potential instability of the Higgs potential (a flat direction for $h_{1}=h_{2}^{*}$ ), which has to be removed by radiative corrections (essentially due to the large top Yukawa coupling). Explicit global symmetry breaking terms in these models lift this flat direction and remove the instability. However, these symmetry breaking terms at the same time invalidate the specific prediction of the 'Higgs as PGB' scheme (the $\mu$-term is not related to soft SUSY breaking mass term anymore), and we will be left with the general Higgs potential of the MSSM. 
In the above model all fields (except $S$ and $N$ ) had the same order of VEV's, thus there is no hierarchy between the $H, \bar{H}$ and $\Sigma$ VEV's. However such a hierarchy may be an attractive feature for generating fermion masses and is also necessary for the unification of couplings. This can be easily achieved in this model by modifying the discrete charges of $\bar{H} H$. We take the $Z_{3}^{(1)} \otimes Z_{3}^{(2)} \otimes Z_{4} \otimes R$ charges for the $\bar{H} H$ as $0, \frac{1}{12}, \frac{1}{4}, \frac{1}{18}$ instead of the charges listed in table 1 (and all other charges are unchanged). Then the only change will be that instead of $S(\bar{H} H)^{2}$ we have $S(\bar{H} H)^{4}$ appearing in the superpotential. This will result in an $H \mathrm{VEV}$ that is the geometric mean value of $M_{P l}$ and $M_{G U T}$, which is desirable for fermion masses. The mixing terms again yield $\mathcal{O}(1000 \mathrm{GeV})$ PGB masses.

\subsection{Model 3}

In the third model we assume that some SU(6) singlet fields have VEV's of the order of the GUT scale through some unspecified dynamics.

One possibility to suppress mixing terms is to have at least two fields whose VEV's are naturally zero in the supersymmetric limit and whose presence is required in all dangerous mixing terms. In this case the mixing terms have the form $S T(\bar{H} H)^{a} \Sigma^{b}$ where $S, T$ are the fields with vanishing VEV's. Then these mixing terms do not contribute to the Higgs masses because of $\langle S\rangle=\langle T\rangle=0$ (if we add the soft breaking terms, $\langle S, T\rangle$ will be of $\mathcal{O}\left(M_{\text {weak }}\right)$, so the contribution to the Higgs masses will be also suppressed by a factor of $M_{\text {weak }} / M_{G U T}$ which is exactly what we need). Thus such fields with vanishing VEV's can yield the desired suppression of the mixing terms.

One such an example could be a superpotential of the form

$$
a S\left(\bar{H} H-\alpha M^{2}\right)+b T\left(\operatorname{Tr} \Sigma^{3}-\beta N^{3}\right) .
$$

(We take a cubic term in $\Sigma$ because the trace of $\Sigma$ vanishes and a quadratic term would give $\mathrm{SU}(35)$ accidental symmetry. $N$ and $M$ should be singlets with respect to the $\mathrm{SU}(6)$ group so that their VEV doesn't break the symmetry further and also to avoid a larger accidental symmetry.)

The equation of motion for the $S, T$ singlets sets

$$
\begin{aligned}
\langle\bar{H} H\rangle & =\alpha\left\langle M^{2}\right\rangle \\
\operatorname{Tr}\left\langle\Sigma^{3}\right\rangle & =\beta\left\langle N^{3}\right\rangle,
\end{aligned}
$$


while the $S$ and $T$ VEV's vanish because of the other equations of motion.

The problem with this model is that the VEV's of the fields $M, N$ and consequently of $\Sigma, H, \bar{H}$ are not determined. To find an acceptable theory based on the superpotential of eq. 4.36 we need to reintroduce the GUT-scale into our theory by setting the $M$ and $N$ VEV's to the GUT-scale by hand. The origin of this new scale in the theory could be for example a condensation scale of a strongly interacting gauge group (other than the $\mathrm{SU}(6)$ ). We assume that for some reason the fields $M, N$ acquire VEV's of $\mathcal{O}\left(M_{G U T}\right)$. Then these VEV's can be communicated to the fields $\Sigma, H, \bar{H}$ without introducing mixing terms through the eqs. 4.37. (In other words we could say that an effective tadpole term in the superpotential for the fields $S$ and $T$ is generated by integrating out heavy fields that have VEV's of the order of the GUT scale which spontaneously break the discrete symmetry.) But even if we set the $M, N$ VEV's to the desired value the $H, \bar{H}, \Sigma$ VEV's are still not totally determined. This is done by the D-terms and the soft breaking terms. The D-terms vanish if $\langle H\rangle=\langle\bar{H}\rangle$ and $\langle\Sigma\rangle$ is diagonal. Now adding the soft breaking terms will shift the values of the VEV's by terms of the order of the weak scale and also lifts the very high degeneracy of the $\Sigma$ vacua. Eq. 4.37 fixes only $\operatorname{Tr} \Sigma^{3}$. After we add the soft breaking terms the only possible $\Sigma$ vacua are those which break $\mathrm{SU}(6)$ to $\mathrm{SU}(\mathrm{n}) \otimes \mathrm{SU}(6-\mathrm{n}) \otimes \mathrm{U}(1)$ depending on the values of the parameters of the soft breaking terms.

To forbid the direct mixing terms (those without the fields $S, T$ ) we should set the discrete charges of $\Sigma, H \bar{H}$ to be small, so that the mixing terms require high powers of these fields. If the discrete symmetry is not an R-type then by choosing the charges of $\Sigma, H \bar{H}$ the charges of the other fields are already determined. For example if we take the charges as $Q_{S}=\frac{15}{16}$, $Q_{\bar{H} H}=Q_{M^{2}}=\frac{1}{16}, Q_{T}=\frac{20}{21}$ and $Q_{\Sigma^{3}}=Q_{N^{3}}=\frac{1}{21}$, the first mixing term without $S, T$ is $(\bar{H} H)^{16} \Sigma^{63}$, or $\bar{H} H, \Sigma$ exchanged to $M^{2}$ or $N$, while the mixing terms involving $S, T$ are automatically suppressed by a factor $\left(\frac{M_{\text {weak }}}{M_{G U T}}\right)$. (These mixing terms are just the products of the operators in the two sectors.)

We can go further and forbid even the mixing terms that include $S, T$ by promoting the discrete symmetry to an R-symmetry. For example if we assign the R-charge for the fields $Q_{S}=\frac{1}{55}, Q_{\bar{H} H}=\frac{2}{165}=Q_{N^{2}}, Q_{T}=\frac{1}{44}, Q_{\Sigma}=Q_{M}=\frac{1}{396}$, and that of the superpotential is $\frac{1}{33}$ then all mixing is forbidden to more than 50 orders. 


\subsection{Summary}

In this section we have presented three different type of models that all yield acceptable theories. We have shown how to circumvent the difficulties of section 3 and build natural theories with sufficiently suppressed mixing terms.

One might think that the above arguments for building a superpotential are true only for the SU(6) model we have considered. In the next section we show that alternative models based on the idea of two noninteracting sectors and either an $\mathrm{SU}(\mathrm{n}), \mathrm{SO}(\mathrm{n})$, or $\mathrm{E}_{6}$ gauge group which do not have additional light doublet or triplet fields are trivial generalizations of the model we have considered, and therefore yield no more compelling solutions. (The only restrictive requirement we will make for this proof is that during SSB the unbroken subgroups are only in regular embeddings of the full group).

\section{$5 \quad$ Models with Larger Gauge Symmetry}

In this section we discuss the possibilities of generalizing the $\mathrm{SU}(6)$ model based on the groups $\mathrm{SU}(\mathrm{n}), \mathrm{SO}(\mathrm{n})$ and $\mathrm{E}_{6}$ (these include all groups that are capable of admitting complex representations). We restrict our search to models that use regular group embeddings only and have no light triplets which could result in too large proton decay.

Let us first discuss the criteria that a realistic model where the Higgses are pseudoGoldstone bosons have to fulfill. We are looking for grand unified models with gauge group $G$ that have an accidental symmetry of the Higgs part of the superpotential. This accidental symmetry is a consequence of the existence of two sectors (A and B) that are not mixed with each other; thus the global symmetry is $G \otimes G$. We do not consider the other kind of models when representations pair up to representations of a bigger group since these models are necessarily fine tuned; a larger numbers of sectors is overly cumbersome and we do not consider it. Throughout this chapter (and Appendix A) we consider only models where the unbroken subgroups of the full symmetry group are in regular embeddings (it is very difficult to derive general results if we also allow the use of special embeddings). The fields in sectors A and B develop VEV's such that the gauge group $G$ breaks to the standard model group $\mathrm{SU}(3) \otimes \mathrm{SU}(2) \otimes \mathrm{U}(1)$. We are interested in all possible symmetry breaking patterns for $G=\mathrm{SU}(\mathrm{n}), \mathrm{SO}(\mathrm{n})$ and $\mathrm{E}_{6}$ for which the resulting uneaten PGB's fulfill the following four requirements. 
1. At least two $\mathrm{SU}(2)$ doublets are uneaten PGB's of the global symmetry which can be identified with the Higgs fields of the MSSM.

2. The extra uneaten PGB's besides the two doublets (if there are some) do not destroy the successful prediction of unification of coupling constants. We know that generally adding extra light particles to the MSSM spectrum destroys the prediction for $\sin ^{2} \theta_{W}$. The only case when this does not happen is when we add full $\mathrm{SU}(5)$ multiplets. Then the one loop value of $\sin ^{2} \theta_{W}$ is unchanged.

3. The extra uneaten PGB's do not give rise to too fast proton decay. This can be prevented if the extra light fields do not contain $\mathrm{SU}(3)$ triplets whose quantum number are equal to those of $d^{c}$ ( $d$ denotes the down quark), because this is the field that if light generically mediates proton decay. Although generally the presence of such light triplets spoils proton decay, we can avoid this problem by forbidding the coupling of these new triplets to ordinary matter (the phenomenological implications of such new triplets were given in [9]). Nevertheless we find it natural to consider only models that do not contain extra $d^{c}$ 's (that is they do not contain for example extra 5's of SU(5)).

4. The extra uneaten PGB's do not destroy asymptotic freedom of QCD. For example if the extra particles are only in $\mathrm{SU}(3)$ triplets than their number is constrained to be less than six. Thus for example the extra uneaten PGB's can not be combined into $10+\overline{10}$ of $\mathrm{SU}(5)$ because this contains exactly 6 triplets.

Summarizing the requirements on the uneaten PGB's for a realistic model we find that in addition to the 2 doublets that are identified with the Higgses of the MSSM we can have only full SU(5) multiplets that do not contain triplets with the quantum numbers of $d^{c}$ and the number of extra triplets is constrained to be less than six.

Let us now discuss how many uneaten PGB's we get from the SSB in the case of $G=\mathrm{SU}(\mathrm{n})$. The general method was presented in [9]. The accidental global symmetry is $\mathrm{SU}(\mathrm{n}) \otimes \mathrm{SU}(\mathrm{n})$. We have two sectors of the superpotential that are not mixed (that's how we get the accidental global $\mathrm{SU}(\mathrm{n}) \otimes \mathrm{SU}(\mathrm{n})$ symmetry). We denote these sectors A and B. The fields in sectors A and B develop VEV's such that the gauged SU(n) breaks to $\mathrm{SU}(3) \otimes \mathrm{SU}(2) \otimes \mathrm{U}(1)$. We are interested in all possible SSB patterns which fulfill the above four requirements of a realistic model and the additional requirement of the use of regular embeddings only.

The VEV's of the fields in one of the two sectors (for example A) have to split the $\mathrm{SU}(3)_{c}$ 
from the $\mathrm{SU}(2)_{w}$ which are embedded into the $\mathrm{SU}(\mathrm{n})$ gauge group. (In the $\mathrm{SU}(6)$ model sector $\mathrm{A}$ is identified with the sector containing the adjoint field. The adjoint in the SU(6) model breaks $\mathrm{SU}(6)$ to $\mathrm{SU}(4) \otimes \mathrm{SU}(2) \otimes \mathrm{U}(1)$ and thus splits the $\mathrm{SU}(3)_{c}$ subgroup from the $\left.\mathrm{SU}(2)_{w} \cdot\right)$

If we consider only regular embeddings of subgroups then the most general subgroups of $\mathrm{SU}(\mathrm{n})$ are products of $\mathrm{SU}(\mathrm{k})$ factors and $\mathrm{U}(1)$ factors. Thus the fields of sector A break the global $\mathrm{SU}(\mathrm{n})$ to

$$
\mathrm{SU}\left(\mathrm{m}_{1}\right) \otimes \mathrm{SU}\left(\mathrm{m}_{2}\right) \otimes \mathrm{SU}\left(\mathrm{m}_{3}\right) \otimes \ldots \otimes \text { possible } \mathrm{U}(1) \text { factors }
$$

where we can assume that $\mathrm{SU}(2)_{\text {weak }} \subset \mathrm{SU}\left(\mathrm{m}_{1}\right), \mathrm{SU}(3)_{c} \subset \mathrm{SU}\left(\mathrm{m}_{2}\right)$. So the VEV's of the fields of this sector split the $\mathrm{SU}(3)_{c}$ subgroup from the $\mathrm{SU}(2)_{w}$ subgroup. Because of our choice of embeddings for the $\mathrm{SU}(2)_{w}$ and $\mathrm{SU}(3)_{c}$ we know that $\mathrm{m}_{1} \geq 2, \mathrm{~m}_{2} \geq 3$ and also $\sum_{i=1}^{l} \mathrm{~m}_{i} \leq \mathrm{n}$ must be true.

The combination of the VEV's of the fields of sectors A and B must break the gauge group to $\mathrm{SU}(3) \otimes \mathrm{SU}(2) \otimes \mathrm{U}(1)$, this means that the gauged subgroup $\mathrm{SU}\left(\mathrm{m}_{1}\right) \subset \mathrm{SU}(\mathrm{n})$ breaks to $\mathrm{SU}(2)$, the $\mathrm{SU}\left(\mathrm{m}_{2}\right) \subset \mathrm{SU}(\mathrm{n})$ breaks to $\mathrm{SU}(3)$, and all other subgroups must be totally broken.

Let us first concentrate on the PGB's we get from the $\mathrm{SU}\left(\mathrm{m}_{1}+\mathrm{m}_{2}\right)$ subgroup of $\mathrm{SU}(\mathrm{n})$ (this is where the standard model group is also embedded). According to our assumptions, the fields of sector A break this $\mathrm{SU}\left(\mathrm{m}_{1}+\mathrm{m}_{2}\right)$ subgroup to $\mathrm{SU}\left(\mathrm{m}_{1}\right) \otimes \mathrm{SU}\left(\mathrm{m}_{2}\right)($ possibly $\otimes \mathrm{U}(1))$, and the combination of the VEV's of both sectors must give $\mathrm{SU}(3) \otimes \mathrm{SU}(2) \otimes \mathrm{U}(1)$ as the unbroken gauge group. This can be achieved only if the fields of sector B break the $\mathrm{SU}\left(\mathrm{m}_{1}+\mathrm{m}_{2}\right)$ subgroup either to $\mathrm{SU}(5)$ or to $\mathrm{SU}(3) \otimes \mathrm{SU}(2) \otimes \mathrm{U}(1)$. First we calculate how many uneaten PGB's we get from this $\mathrm{SU}\left(\mathrm{m}_{1}+\mathrm{m}_{2}\right)$ subgroup if the fields of sector B break this $\mathrm{SU}\left(\mathrm{m}_{1}+\mathrm{m}_{2}\right)$ subgroup to SU(5). Uneaten PGB's are states in the SU(n) adjoint corresponding to generators that are broken by the fields of both sectors. It is easy to check that from the breaking $\mathrm{SU}\left(\mathrm{m}_{1}+\mathrm{m}_{2}\right) \otimes \mathrm{SU}\left(\mathrm{m}_{1}+\mathrm{m}_{2}\right) \rightarrow \mathrm{SU}\left(\mathrm{m}_{1}\right) \otimes \mathrm{SU}\left(\mathrm{m}_{2}\right) \otimes \mathrm{SU}(5)$ we get the following uneaten PGB's:

$$
\left(\mathrm{m}_{1}-3\right)\left[(1,2)_{\frac{1}{2}}+(1,2)_{-\frac{1}{2}}\right]+\left(\mathrm{m}_{2}-2\right)\left[(3,1)_{-\frac{2}{3}}+(\overline{3}, 1)_{\frac{2}{3}}\right]+\text { singlets },
$$

where the PGB's are denoted according to their $\mathrm{SU}(3) \otimes \mathrm{SU}(2) \otimes \mathrm{U}(1)$ transformation properties. (If fields of sector B break $\mathrm{SU}\left(\mathrm{m}_{1}+\mathrm{m}_{2}\right)$ to $\mathrm{SU}(3) \otimes \mathrm{SU}(2) \otimes \mathrm{U}(1)$ instead of $\mathrm{SU}(5)$ we get extra uneaten PGB's in the representations $(3,2)+(\overline{3}, 2)$ of $\mathrm{SU}(3) \otimes \mathrm{SU}(2)$. These representations contain 6 doublets, so even if we combine them with some other light particles 
to full $\mathrm{SU}(5)$ representations (to preserve the prediction for $\sin ^{2} \theta_{W}$ ) we will have at least 6 extra triplets added to the MSSM particle content. This is unacceptable because of the asymptotic freedom of QCD. Thus the fields of sector B must break the $\mathrm{SU}\left(\mathrm{m}_{1}+\mathrm{m}_{2}\right)$ subgroup to $\mathrm{SU}(5)$.) To avoid the presence of triplets in eq. 5.2 (these triplets are contained in 5 and $\overline{5}$ 's of $\mathrm{SU}(5)$ so they generally give rise to fast proton decay) we need $\mathrm{m}_{2}=2$. Because from the states outside the $\mathrm{SU}\left(\mathrm{m}_{1}+\mathrm{m}_{2}\right)$ subgroup we can get only full $\mathrm{SU}(5)$ representations as uneaten PGB's we need to set $\mathrm{m}_{1}=4$ to get the two light uneaten doublets. Thus $\mathrm{m}_{1}=4, \mathrm{~m}_{2}=2$, and the fields of sector A break this $\mathrm{SU}\left(\mathrm{m}_{1}+\mathrm{m}_{2}\right)$ subgroup (which is an $\mathrm{SU}(6)$ because $\left.\mathrm{m}_{1}=2, \mathrm{~m}_{2}=4\right)$ to $\mathrm{SU}(4) \otimes \mathrm{SU}(2) \otimes \mathrm{U}(1)$ and the fields of sector $\mathrm{B}$ to $\mathrm{SU}(5)$.

Now we are able to discuss the SSB of the full global SU(n) $\otimes \mathrm{SU}(\mathrm{n})$. According to our assumptions and the previous argument, the fields of sector A break $\mathrm{SU}(\mathrm{n})$ to $\mathrm{SU}(4) \otimes \mathrm{SU}(2)$ $\otimes \mathrm{SU}\left(\mathrm{m}_{3}\right) \otimes \ldots \otimes \mathrm{U}(1)$ factors. This means that the generators in the two off-diagonal 5 by $(\mathrm{n}-6)$ matrices of the $\mathrm{SU}(\mathrm{n})$ adjoint are already broken by the fields of sector $\mathrm{A}$, and all these states transform nontrivially under $\mathrm{SU}(3) \otimes \mathrm{SU}(2) \otimes \mathrm{U}(1)$ of the MSSM. If we suppose that the fields of sector B break $\mathrm{SU}(\mathrm{n})$ to a smaller subgroup than $\mathrm{SU}(\mathrm{n}-1)$ then some of these states (in the offdiagonal 5 by $n-6$ matrices of the adjoint) were also uneaten PGB's and we would get additional light doublets and triplets. These states transform all according to 5 or $\overline{5}$ of SU(5). Thus they would give rise to proton decay. This means that the only acceptable breaking for the fields of sector B is to break $\mathrm{SU}(\mathrm{n})$ to $\mathrm{SU}(\mathrm{n}-1)$. But because the unbroken gauge group is $\mathrm{SU}(3) \otimes \mathrm{SU}(2) \otimes \mathrm{U}(1)$ and we get this by combining the VEV's of both sectors we must have $\mathrm{m}_{i}=0$ for $i>2$ in sector $\mathrm{A}$. Otherwise we would get a bigger unbroken gauge group than $\mathrm{SU}(3) \otimes \mathrm{SU}(2) \otimes \mathrm{U}(1)$. This means that for sector $\mathrm{A}, \mathrm{SU}(\mathrm{n}) \rightarrow \mathrm{SU}(4) \otimes \mathrm{SU}(2) \otimes \mathrm{U}(1)$, while for sector $\mathrm{B}, \mathrm{SU}(\mathrm{n}) \rightarrow \mathrm{SU}(\mathrm{n}-1)$.

Thus we have shown that if we rely only on regular embeddings of subgroups into bigger groups the only acceptable pattern of symmetry breaking in $\mathrm{SU}(\mathrm{n})$ models is $\mathrm{SU}(\mathrm{n}) \otimes$ $\mathrm{SU}(\mathrm{n}) \rightarrow \mathrm{SU}(4) \otimes \mathrm{SU}(2) \otimes \mathrm{U}(1) \otimes \mathrm{SU}(\mathrm{n}-1)$. This generates two light doublets and additional light $\mathrm{SU}(3) \otimes \mathrm{SU}(2) \otimes \mathrm{U}(1)$ singlets. This is an obvious generalization of the $\mathrm{SU}(6)$ model, and we have also proven that there is no other way in $\mathrm{SU}(\mathrm{n})$ models using only regular embeddings to implement the idea of having the Higgses as PGB's. Using the same requirements we show in Appendix A that there is no satisfactory model based on the gauge groups $\mathrm{SO}(\mathrm{n})$ or $\mathrm{E}_{6}$. Thus we see that all realistic models that have the Higgses as PGB's and which do not require special embeddings are based on the unification group $\mathrm{SU}(\mathrm{n})$, and for every 
$\mathrm{n}$ there is only one possibility for the pattern of symmetry breaking.

Finally we give the necessary fields for the generalized $\mathrm{SU}(\mathrm{n})$ theories with $\mathrm{n} \geq 6$, where the symmetry breaking pattern of the accidental global symmetry is $\mathrm{SU}(\mathrm{n}) \otimes \mathrm{SU}(\mathrm{n}) \rightarrow \mathrm{SU}(4)$ $\otimes \mathrm{SU}(2) \otimes \mathrm{U}(1) \otimes \mathrm{SU}(\mathrm{n}-1)$. This symmetry breaking can be most naturally achieved with the following fields: an adjoint $(\Sigma)$ and $\mathrm{n}-6$ pairs of vectors $\left(H_{i}, \bar{H}_{i}\right)$ in sector A of the superpotential and another pair of vectors $\left(H^{\prime}, \bar{H}^{\prime}\right)$ in sector B with the following VEV's:

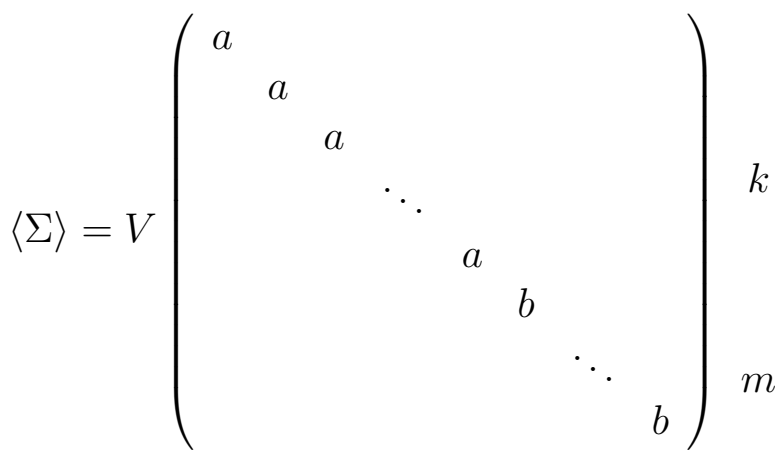

$$
\begin{aligned}
& \left\langle H_{i}\right\rangle=\left\langle\bar{H}_{i}\right\rangle=V_{i}\left(\begin{array}{c}
0 \\
0 \\
\vdots \\
1 \\
\vdots
\end{array}\right) \\
& \left\langle H^{\prime}\right\rangle=\left\langle\bar{H}^{\prime}\right\rangle=W\left(\begin{array}{c}
1 \\
0 \\
0 \\
\vdots \\
0
\end{array}\right)
\end{aligned}
$$

with $k a+m b=0, W>V, V_{i}$ (in order to get the correct order of gauge symmetry breaking $\mathrm{SU}(\mathrm{n}) \rightarrow \mathrm{SU}(\mathrm{n}-1) \rightarrow \mathrm{SU}(3) \otimes \mathrm{SU}(2) \otimes \mathrm{U}(1))$, the smallest of $V, V_{i}$ must be the GUT scale, and the ones in the VEV's of $V_{i}$ are organized such that together with the adjoint they break $\mathrm{SU}(\mathrm{n})$ to $\mathrm{SU}(4) \otimes \mathrm{SU}(2) \otimes \mathrm{U}(1)$.

The minimal anomaly free fermion content of an $\mathrm{SU}(\mathrm{n})$ theory [15] is one two index antisymmetric representation (denoted by $T^{a b}$ ) and $n-4$ conjugates of the defining representation $\left(t_{a}\right)$ per family. If we only want to have the usual light $\mathrm{SU}(5)$ fermions $(10+\overline{5})_{i}$, 
then the remaining $\mathrm{SU}(5)$ nonsinglet fields must be heavy. The $\mathrm{SU}(5)$ content of $T^{a b}$ and $t_{a}$ is

$$
\begin{aligned}
& T^{a b}=10+(\mathrm{n}-5) 5+\text { singlets } \\
& t_{a}=\overline{5}+\text { singlets }
\end{aligned}
$$

This means that in the case of 3 families we must have $3(\mathrm{n}-5)$ heavy 5 's and $\overline{5}$ 's.

Operators that could give GUT-scale masses to fields of eq. 5.6 are:

$$
\begin{aligned}
& \lambda_{i j} T_{(i)}^{a b} t_{a(j)} \bar{H}_{b} \\
& \lambda_{i j}^{m} T_{(i)}^{a b} t_{a(j)} \bar{H}_{b}^{\prime m}
\end{aligned}
$$

where $i, j$ denote generation indices while $a, b$ denote $\mathrm{SU}(\mathrm{n})$ indices. The first term yields 3 heavy 5's and 5's while the second term yields $3(\mathrm{n}-6)$ heavy 5 's and $\overline{5}$ 's.

Thus it is possible to split the heavy fields from the light in a natural way for every $\mathrm{n}$. The bigger problem is to account for a heavy $\left(\mathcal{O}\left(M_{W}\right)\right.$ ) top quark. The method of [13 (see also Appendix B) does not work for $n>6$. In this case the smallest self adjoint representation that does not have a heavy mass term is a representation with $\mathrm{n} / 2$ antisymmetrized indices for $\mathrm{n}$ even but $\mathrm{n} / 2$ odd. But the $\mathrm{SU}(5)$ content of this field (called $M$ ) is

$$
M=\left(\begin{array}{c}
n-5 \\
\frac{n}{2}-1
\end{array}\right)(5+\overline{5})+\left(\begin{array}{c}
n-5 \\
\frac{n}{2}-2
\end{array}\right)(10+\overline{10})+\text { singlets. }
$$

out of which we only want to have one of the 10's light, and all other fields must be heavy (and also one of the 10's of $T^{a b}$ must then be heavy). We show that with these fields we can not have a consistent theory. The reason is that with only these fields and assuming matter parity we can have only one renormalizable operator containing $M$ in the superpotential, $M \Sigma M$. But this does not give GUT-scale mass to any of the fields in $M$ because of the antisymmetry of the indices. (In the case of SU(6) there was another operator $20 H 15$ that made the extra unwanted fields heavy, but in the case $\mathrm{n}>6$ the operator $M H_{i} T$ is not $\mathrm{SU}(\mathrm{n})$ invariant). Thus the masses for these new fields coming from $M$ can arise only from the nonrenormalizable operators. But this means that all the fields from $M$ have masses less than the GUT-scale. Because $M$ is a very big representation of $\mathrm{SU}(\mathrm{n})$ it contains a lot of new triplets. (The lowest possible value of $n$ is 10 , because this is the first case when $n$ 
is even and $n / 2$ is odd. If $n=10$, the extra number of triplets is 70 .) This badly destroys asymptotic freedom and is therefore not acceptable (in the case of $n=10$ the couplings blow up before we reach $M_{G U T}$, and the $\mathrm{n}>10$ cases are even worse). Also because we don't have renormalizable mass terms for the fields in $M$ the masses of the triplets will be lower than $M_{G U T}$ which is dangerous for proton decay.

It is even more difficult to find an acceptable superpotential for the $\mathrm{n}>6$ models than in the $n=6$ case. The extra difficulty comes from the sector where we need to combine an adjoint and $n-6$ pairs of vectors to get the required symmetry breaking. In this sector we have to mix the adjoint and the vectors to avoid a bigger accidental symmetry than $\mathrm{SU}(\mathrm{n}) \otimes \mathrm{SU}(\mathrm{n})$. Thus we necessarily have terms of the form $\bar{H}_{i} \Sigma H_{j}$. But the presence of such terms generally requires $\langle H\rangle=0$. We illustrate this for the a superpotential with $\mathrm{SU}(6)$ fields:

$$
W=\frac{1}{2} M \operatorname{Tr} \Sigma^{2}+\frac{1}{3} \lambda \operatorname{Tr} \Sigma^{3}+\alpha \bar{H} \Sigma H .
$$

The equation of motion for $\Sigma$ will be

$$
M \Sigma_{i j}+\lambda\left(\Sigma_{i j}^{2}-\frac{1}{6} \operatorname{Tr} \Sigma^{2} \delta_{i j}\right)+\alpha \bar{H}_{i} H_{j}=0 .
$$

If we assume that

$$
\langle\Sigma\rangle=V\left(\begin{array}{cccccc}
1 & & & & & \\
& 1 & & & & \\
& & 1 & & & \\
& & & 1 & & \\
& & & & -2 & \\
& & & & & -2
\end{array}\right),\langle H\rangle=\langle\bar{H}\rangle=W\left(\begin{array}{l}
1 \\
0 \\
0 \\
0 \\
0 \\
0
\end{array}\right),
$$

we get for the $\Sigma$ equation of motion that

$$
\left(M V+\lambda V^{2}\right)\left(\begin{array}{cccccc}
1 & & & & & \\
& 1 & & & & \\
& & 1 & & & \\
& & 1 & & \\
& & & -2 & \\
& & & & & -2
\end{array}\right)+\alpha W^{2}\left(\begin{array}{ccccc}
1 & & & & \\
& 0 & & & \\
& & 0 & & \\
& & 0 & & \\
& & & 0 & \\
& & & & 0
\end{array}\right)=0
$$

which in turn requires that $W=0$. Thus it is difficult to find a superpotential where adjoints and vectors are mixed and both have comparable VEV's. (We need to introduce extra fields that mix with both the adjoint and the vectors, but which have zero VEV's while they allow for nonzero VEV's of both the adjoint and the vectors.) 


\section{Conclusions}

The biggest problem of the SU(6) model where the Higgses are PGB's of the spontaneously broken accidental $\mathrm{SU}(6) \otimes \mathrm{SU}(6)$ symmetry is to construct a superpotential with some possible discrete symmetries that yields naturally the correct VEV's for the fields and mixing is forbidden to sufficiently high order. We have shown how to construct such models. We have also shown why we expect these to be the only type of models which will work. The reason for this is that without having either a small mass scale or fields with zero VEV in our theory we generally need either fine tuning of the parameters or have the symmetry breaking mixing terms not sufficiently suppressed. We have demonstrated that we can naturally make use of the mass scales present in a theory to build an acceptable model. We have also shown that we can build models without using small mass scales by using fields that have naturally zero VEV's. We presented three possible models with sufficiently suppressed mixing terms. In the first example we made use of the supersymmetry breaking scale that is necessarily present in every theory. In the second model we did not use small mass scales but had singlets with zero VEV's. In the third model we assumed the dynamical appearance of the GUT scale.

We have also shown that there are only very few realistic models that use only regular group embeddings and have the Higgses as PGB's. All of them are trivial generalizations of the $\mathrm{SU}(6)$ model built on the gauge group $\mathrm{SU}(\mathrm{n})$.

We conclude that it is possible to successfully implement the Higgs as Goldstone boson scheme in models where only discrete global symmetries or gauge symmetries are assumed to be exact to all orders. Based on our considerations we expect that the simplest models successfully implementing the idea of having the Higgses as pseudo-Goldstone particles will have features of those presented in section 4 .

\section{Acknowledgements}

C.C. and L.R. are grateful to Greg Anderson for useful discussions and especially for pointing out the fine tuning in ref. 12]. Z.B. thanks Oleg Kancheli for useful discussions. Z.B. and L.R. thank S. Pokorsky and the other organizers for their hospitality at the Warsaw SUSY Meeting 'Physics from Planck Scale to Electroweak Scale', where part of this work was done. 


\section{Appendix A: $\mathrm{SO}(\mathbf{n})$ and $\mathrm{E}_{6}$}

In this appendix we show that it is not possible to generalize the $\mathrm{SU}(6)$ model to orthogonal groups or to $\mathrm{E}_{6}$ if one allows only for regular embeddings.

If the gauge group is $\mathrm{SO}(\mathrm{n})$ then the $\mathrm{SU}(2)_{w}$ must be embedded into an $\mathrm{SO}(4)$ subgroup and the $\mathrm{SU}(3)_{c}$ into an $\mathrm{SO}(6)$ subgroup of $\mathrm{SO}(\mathrm{n})$, thus

$$
\mathrm{SU}(3) \otimes \mathrm{SU}(2) \subset \mathrm{SO}(6) \otimes \mathrm{SO}(4) \subset \mathrm{SO}(10)
$$

We know that $\mathrm{SO}(6) \sim \mathrm{SU}(4), \mathrm{SO}(4) \sim \mathrm{SU}(2) \otimes \mathrm{SU}(2)$, so the $\mathrm{SU}(3) \otimes \mathrm{SU}(2)$ transformation properties of the states in the adjoint (45) of the $\mathrm{SO}(10)$ subgroup of $\mathrm{SO}(\mathrm{n})$ where the standard model group is embedded are

$$
\begin{aligned}
& {[(8,1)+(3,1)+(\overline{3}, 1)+(1,1)]+} \\
& {[(1,3)+3(1,1)]+2[(3,2)+(\overline{3}, 2)]+\text { singlets. }}
\end{aligned}
$$

Now we can apply the same method we used for $\mathrm{SU}(\mathrm{n})$. We have again two sectors, A and B. The VEV's of the fields of one of the sectors $(\mathrm{A})$ have to split the $\mathrm{SU}(2)_{w}$ and $\mathrm{SU}(3)_{c}$ subgroups, and thus $\mathrm{SO}(\mathrm{n})$ breaks either to $\mathrm{SO}\left(\mathrm{m}_{1}\right) \otimes \mathrm{SO}\left(\mathrm{m}_{2}\right) \otimes \ldots$ or to $\mathrm{SU}\left(\mathrm{n}_{1}\right) \otimes \mathrm{SU}\left(\mathrm{n}_{2}\right) \otimes \ldots$. In the first case (orthogonal subgroups) we have the embedding $\mathrm{SU}(3) \subset \mathrm{SO}\left(\mathrm{m}_{1}\right), \mathrm{SU}(2) \subset$ $\mathrm{SO}\left(\mathrm{m}_{2}\right)$. The combination of the VEV's of the two sectors must break the gauged $\mathrm{SO}\left(\mathrm{m}_{1}\right)$ to $\mathrm{SU}(3)$ and the gauged $\mathrm{SO}\left(\mathrm{m}_{2}\right)$ to $\mathrm{SU}(2)$. If the fields of the sector A break the $\mathrm{SO}\left(\mathrm{m}_{1}+\mathrm{m}_{2}\right)$ subgroup to $\mathrm{SO}\left(\mathrm{m}_{1}\right) \otimes \mathrm{SO}\left(\mathrm{m}_{2}\right)$, then we need the fields of sector B to break the $\mathrm{SO}\left(\mathrm{m}_{1}+\mathrm{m}_{2}\right)$

subgroup to $\mathrm{SU}(5)$ so that the combination of the VEV's of the two sectors break the gauged $\mathrm{SO}\left(\mathrm{m}_{1}+\mathrm{m}_{2}\right)$ to $\mathrm{SU}(3) \otimes \mathrm{SU}(2) \otimes \mathrm{U}(1)$. (Should we choose $\mathrm{SU}(3) \otimes \mathrm{SU}(2) \otimes \mathrm{U}(1)$ instead of $\mathrm{SU}(5)$ for the breaking of $\mathrm{SO}\left(\mathrm{m}_{1}+\mathrm{m}_{2}\right)$ by the fields of sector B we would get more PGB's just like in the case of $\mathrm{SU}(\mathrm{n})$ models). One can show that from this $\mathrm{SO}\left(\mathrm{m}_{1}+\mathrm{m}_{2}\right)$ subgroup alone we get the following PGB's:

$$
\left(\mathrm{m}_{1}-6\right) 2(1,2)+\left(\mathrm{m}_{2}-4\right)[(3,1)+(\overline{3}, 1)]+(3,2)+(\overline{3}, 2)
$$

where the PGB's are denoted according to their $\mathrm{SU}(3) \otimes \mathrm{SU}(2)$ transformation properties. So no matter what $m_{1}$ and $m_{2}$ are we get the light $(3,2)+(\overline{3}, 2)$ particles which are unacceptable because they destroy unification (if we want them to combine with some other light states 
to full SU(5) representations, we get at least 6 triplets because we already have 6 doublets. Thus in this case asymptotic freedom of QCD would be destroyed).

The other possibility is that $\mathrm{SU}(3) \otimes \mathrm{SU}(2)$ is embedded in unitary groups in sector A, $\mathrm{SU}(3) \otimes \mathrm{SU}(2) \subset \mathrm{SU}\left(\mathrm{m}_{1}\right) \otimes \mathrm{SU}\left(\mathrm{m}_{2}\right) \subset \mathrm{SO}\left(2 \mathrm{~m}_{1}\right) \otimes \mathrm{SO}\left(2 \mathrm{~m}_{2}\right) \subset \mathrm{SO}\left(2 \mathrm{~m}_{1}+2 \mathrm{~m}_{2}\right)$. In this case we need to break this $\mathrm{SO}\left(2 \mathrm{~m}_{1}+2 \mathrm{~m}_{2}\right)$ subgroup by the fields of sector B to an $\mathrm{SO}(10)$ subgroup in order to break the gauged $\mathrm{SO}\left(2 \mathrm{~m}_{1}+2 \mathrm{~m}_{2}\right)$ subgroup in the right way. The resulting uneaten PGB's from this $\mathrm{SO}\left(2 \mathrm{~m}_{1}+2 \mathrm{~m}_{2}\right)$ subgroup can be shown to be (according to their $\mathrm{SU}(3) \otimes \mathrm{SU}(2)$ transformation properties):

$$
\begin{aligned}
& \left(2 \mathrm{~m}_{2}+\mathrm{m}_{1}-7\right)[(3,1)+(\overline{3}, 1)]+ \\
& 2\left(2 \mathrm{~m}_{1}+\mathrm{m}_{2}-8\right)(1,2)+\text { singlets }
\end{aligned}
$$

Because $\mathrm{m}_{1} \geq 3, \mathrm{~m}_{2} \geq 2$, we can get zero triplets (they are again part of 5's and 5's of SU(5) so their presence would generally give rise to proton decay) only if $\mathrm{m}_{1}=3$ and $\mathrm{m}_{2}=2$. This just means that $\mathrm{SO}\left(2 \mathrm{~m}_{1}+2 \mathrm{~m}_{2}\right)=\mathrm{SO}(10)$ and the fields of sector A break $\mathrm{SO}(10)$ to $\mathrm{SU}(3) \otimes \mathrm{SU}(2) \otimes \mathrm{U}(1)$, and the fields of sector B do not break this $\mathrm{SO}(10)$ subgroup of $\mathrm{SO}(\mathrm{n})$. But subsequently from the other subgroups we can get only full $\mathrm{SU}(5)$ multiplets, so we do not have doublet PGB's. This means that there is no $\mathrm{SO}(\mathrm{n})$ theory with only regular embeddings that would successfully generalize the $\mathrm{SU}(6)$ model. (If the $\mathrm{SU}(3) \otimes \mathrm{SU}(2)$ are mixed in a unitary and an orthogonal subgroup in sector $\mathrm{A}$ that is $\mathrm{SU}(3) \subset \mathrm{SO}\left(\mathrm{m}_{1}\right)$ and $\mathrm{SU}(2) \subset \mathrm{SU}\left(\mathrm{m}_{2}\right)$ or $\mathrm{SU}(3) \subset \mathrm{SU}\left(\mathrm{m}_{1}\right)$ and $\mathrm{SU}(2) \subset \mathrm{SO}\left(\mathrm{m}_{2}\right)$ we get the same analysis as for the case when $\mathrm{SU}(3) \subset \mathrm{SO}\left(\mathrm{m}_{1}\right)$ and $\mathrm{SU}(2) \subset \mathrm{SO}\left(\mathrm{m}_{2}\right)$ but with even more PGB's, so these cases do not work either.)

In the case of the $\mathrm{E}_{6}$ group we can have 3 possible regular embeddings of $\mathrm{SU}(3) \otimes$ $\mathrm{SU}(2) \otimes \mathrm{U}(1)$ into $\mathrm{E}_{6}$. These cases correspond to the following maximal subgroups of $\mathrm{E}_{6}$ : $\mathrm{SU}(6) \otimes \mathrm{SU}(2), \mathrm{SO}(10) \otimes \mathrm{U}(1)$ or $\mathrm{SU}(3) \otimes \mathrm{SU}(3) \otimes \mathrm{SU}(3)$.

If we place $\mathrm{SU}(3) \otimes \mathrm{SU}(2)$ into the $\mathrm{SU}(6)$ subgroup we can repeat our argument that in one sector the global $\mathrm{SU}(6)$ must break to $\mathrm{SU}(4) \otimes \mathrm{SU}(2) \otimes \mathrm{U}(1)$ while in the other sector to $\mathrm{SU}(5)$. The adjoint of $\mathrm{E}_{6}$ is 78 , its decomposition under $\mathrm{SU}(6) \otimes \mathrm{SU}(2)$ is

$$
78=(35,1)+(1,3)+(20,2)
$$

Because fields of both both sectors break the adjoint along the $\mathrm{SU}(6) \otimes \mathrm{SU}(2)$ subgroup the states in $(20,2)$ must be uneaten PGB's. Their SU(5) decomposition is $2(10+\overline{10})$, and these 
would be light. But this would yield 12 new light triplets that destroy asymptotic freedom of QCD. We also saw that based on orthogonal groups we can not build an acceptable model, so the $\mathrm{SO}(10) \otimes \mathrm{U}(1)$ case is excluded as well.

The last possibility for $\mathrm{E}_{6}$ models is to embed $\mathrm{SU}(3) \otimes \mathrm{SU}(2) \otimes \mathrm{U}(1)$ into the $\mathrm{SU}(3) \otimes$ $\mathrm{SU}(3) \otimes \mathrm{SU}(3)$ subgroup. The decomposition of 78 under this subgroup is

$$
78=(8,1,1)+(1,8,1)+(1,1,8)+(3,3, \overline{3})+(\overline{3}, \overline{3}, 3) .
$$

The fields in one of the sectors (A) need to break $\mathrm{E}_{6}$ at least to $\mathrm{SU}(3) \otimes \mathrm{SU}(3) \otimes \mathrm{SU}(3)$ to split the $\mathrm{SU}(3)_{c}$ from $\mathrm{SU}(2)_{w}$. Because of the unification of couplings this must happen at the GUT scale, and the fields of the other sector (B) must break $\mathrm{E}_{6}$ at a scale above $M_{G U T}$ to either SU(5), $\mathrm{SU}(6)$ or $\mathrm{SO}(10)$. (Otherwise we get large threshold corrections for the $\mathrm{RG}$ value of $\sin ^{2} \theta_{W}$.) If the fields of sector $\mathrm{B}$ break $\mathrm{E}_{6}$ to $\mathrm{SU}(5)$ than only particles in $(3,2)+(\overline{3}, 2)$ will be eaten from sector A which still leaves at least fields transforming according to $\mathrm{SU}(3) \otimes \mathrm{SU}(2)$ as

$$
3[(\overline{3}, 1)+(3,1)]+2[(\overline{3}, 2)+(3,2)]
$$

uneaten. (For the unification of couplings it would be sufficient that fields of sector B break the global $\mathrm{E}_{6}$ to $\mathrm{SU}(5) \otimes$ possible other factor like $\mathrm{SU}(5) \otimes \mathrm{SU}(2)$, but these possible other factors do not play any role since their presence can only change the number of SU(5) singlets. Thus the same analysis would apply. The same is true for the next two cases when the fields of sector B break $\mathrm{E}_{6}$ to $\mathrm{SO}(10)$ or $\mathrm{SU}(6)$.)

If the fields of sector $\mathrm{B}$ break $\mathrm{E}_{6}$ to $\mathrm{SO}(10)$ than one can show that the uneaten PGB's are at least (according to their $\mathrm{SU}(3) \otimes \mathrm{SU}(2)$ transformation properties)

$$
(3,1)+(\overline{3}, 1)+2[(\overline{3}, 2)+(3,2)] .
$$

Finally if the fields of sector $\mathrm{B}$ break $\mathrm{E}_{6}$ to $\mathrm{SU}(6)$ the uneaten nontrivially transforming PGB's are at least

$$
2[(\overline{3}, 1)+(3,1)]+3[(\overline{3}, 2)+(3,2)] .
$$

This means that in neither case are we able to get the desired light particle spectrum (in each case we get more than six triplets), so there is no way to have a realistic model built on $\mathrm{E}_{6}$ that has the Higgs particles as PGB's using these embeddings. 


\section{Appendix B: Fermion Masses}

In this appendix we show that it is possible to extend the successful picture of fermion masses of refs. 13, 14 with the discrete charges in accordance with a realistic Higgs sector. For the Higgs sector we will use the superpotential presented in section 4, extending the discrete symmetry to the fermion sector. We will see a model that is consistent both in the Higgs sector and in the fermion sector. This serves as an existence proof for realistic models implementing the idea of having the Higgs fields as pseudo-Goldstone bosons.

First we briefly review the model of refs. [13, 14] for fermion masses in the SU(6) model. The minimal anomaly free fermion content of SU(6) that includes one generation of light fermions is

$$
15+\overline{6}+\overline{6}^{\prime}
$$

where 15 is the two index antisymmetric representation and $\overline{6}$ is the conjugate of the defining representation. One can add any self adjoint representation and maintain anomaly cancellation. Generally self adjoint representations have invariant mass terms so it is no use adding them to the fermion content. But there are some special cases when this mass term vanishes. For example if we add just one representation 20 of SU(6) (three index antisymmetric representation) then the mass term for this vanishes by antisymmetry (in general if we have odd number of 20's one of them will have a vanishing mass). We remark that the addition of a 20 to the usual particle content of the theory destroys asymptotic freedom of the $\mathrm{SU}(6)$ gauge coupling. But this is not a problem since with only one 20 the increase of the coupling is very slow, its value increases only a few percent between the GUT and the Planck scale.

The idea of [13] is to add the extra 20 to the fermion content which will be then

$$
\left(15+\overline{6}+\overline{6}^{\prime}\right)_{i}+20, \quad i=1,2,3
$$

The SU(5) decomposition of these fields is

$$
\begin{aligned}
& 20=10+\overline{10}, \\
& 15=10+5, \\
& \overline{6}=\overline{5}+1 .
\end{aligned}
$$

Then the renormalizable Yukawa couplings have the form

$$
\lambda^{(1)} 20 \Sigma 20+\lambda^{(2)} 20 H 15_{i}+\lambda_{i j}^{(3)} 15_{i} \bar{H} \overline{6}_{j}^{\prime}, \quad i, j=1,2,3
$$


( $i, j$ denote generation indices). If we insert the VEV's of $H, \bar{H}, \Sigma$ and the Higgs doublets into $\Sigma$ (if $\langle H\rangle \gg\langle\Sigma\rangle$, the Higgs doublets live almost entirely in the $\Sigma$ field, see eqs. 2.6,2.7) we get the following mass terms:

$$
\lambda^{(2)}\langle H\rangle 10_{i} \overline{10}+\lambda_{i j}^{(3)}\langle H\rangle 5_{i} \overline{5}_{j}^{\prime}+\lambda^{(1)} Q u^{c} h_{2}, \quad i, j=1,2,3,
$$

where the decomposition of 10 of $\mathrm{SU}(5)$ is $Q+u^{c}+e^{c}$. The fermion fields in (B.2) contain altogether four 10's, six 5's, three 5's and one $\overline{10}$ of SU(5). From (B.5) we see that out of these fields one combination of 10's, three of $\overline{5}$ 's and the three 5 's and the $\overline{10}$ will get masses of $\mathcal{O}\left(M_{G U T}\right)$, so the light fermion spectrum is the desired

$$
3 \times(10+\overline{5}),
$$

while only one light fermion (namely, the up type quark contained in 20) gets a mass from the renormalizable interaction with the Higgs doublet. The reason is that the couplings of the 20-plet explicitly violate the global $\mathrm{SU}(6)_{\Sigma} \otimes \mathrm{SU}(6)_{H}$ symmetry, so that the Higgs doublet $h_{2}$ has non-vanishing coupling to the up type quark from 20 , which can be identified with the top quark. Thus, the top mass is naturally in the $100 \mathrm{GeV}$ range. Other fermions stay massless at this level, unless we invoke the higher order operators explicitly violating the accidental global symmetry.

To go further we need to introduce nonrenormalizable operators to give masses to the other fermions. Generally, these operators explicitly violate the accidental global symmetry, since they include both the $\Sigma$ and $H, \bar{H}$ fields, so that they can provide nonvanishing Yukawa couplings to the Higgs doublets, though suppressed by $M_{P l}$. In refs. [13, 14 these operators were obtained from heavy fermion exchange [16]. For this purpose a specific set of heavy (Planck-scale) vectorlike fermion superfields in nontrivial SU(6) representations was introduced whose couplings with the light fermions and the Higgs superfields yielded the needed structure of nonrenormalizable operators (together with flavor-blind discrete $Z_{2}$ [13] or $Z_{3}$ [14] symmetries to forbid some unwanted operators). For example, in ref. [13] the relevant nonrenormalizable operators coming from the specified heavy fermion superfield exchanges were specified by

$$
\begin{aligned}
& \frac{1}{M_{P l}}(20 \Sigma) H 15_{i}, i=1,2,3, \\
& \frac{1}{M_{P l}^{2}} 20(\bar{H} \Sigma \bar{H}) \overline{6}_{3}
\end{aligned}
$$




\begin{tabular}{|c|c|c|c|c|c|c|c|c|c|c|c|}
\hline & $H$ & $H$ & $\Sigma_{1}$ & $\Sigma_{2}$ & 20 & $15_{3}$ & $15_{2,1}$ & $\overline{6}_{3,1}$ & $\overline{6}_{2}$ & $\overline{6}_{3}^{\prime}$ & $\overline{6}_{2,1}^{\prime}$ \\
\hline$Z_{4}^{(1)}$ & $\frac{1}{n}$ & 0 & 0 & 0 & 0 & $-\frac{1}{n}$ & $-\frac{1}{n}$ & 0 & $\frac{1}{n}$ & $\frac{1}{n}$ & $\frac{1}{n}$ \\
\hline$Z_{4}^{(2)}$ & 0 & $\frac{1}{n}$ & 0 & 0 & 0 & 0 & 0 & $-\frac{2}{n}$ & $-\frac{1}{n}$ & $-\frac{1}{n}$ & $-\frac{1}{n}$ \\
\hline$Z_{3}$ & 0 & 0 & $\frac{1}{3}$ & $-\frac{1}{3}$ & $\frac{1}{3}$ & $-\frac{1}{3}$ & 0 & 0 & 0 & $\frac{1}{3}$ & 0 \\
\hline
\end{tabular}

Table 2: The charge assignments of the chiral superfields under the discrete $Z_{n}^{(1)} \otimes Z_{n}^{(2)} \otimes Z_{3}$ symmetry.

$$
\frac{1}{M_{P l}^{2}} 15_{i}(\Sigma \bar{H})\left(\Sigma \overline{6}_{j}\right), i, j=2,3 .
$$

The first operator gives mass to the $c$ quark, the second to the $b$ and $\tau$, and the third to the $s$ and $\mu$. These masses will have a proper hierarchy provided that $\langle H\rangle>\rangle\langle\Sigma\rangle$. In the model of ref. 13 the first generation fermions were left massless, however in the model of ref. 14] they can also get masses of the needed value.

However in our approach all nonrenormalizable operators that are not forbidden by some symmetry are present in the superpotential. In other words, we would like to obtain all masses in general operator analysis, not relying on heavy fermion exchange mechanism [16] with specified fields. Therefore a 'flavor democratic' approach to fermion masses (which means that there are no 'family symmetries' that would distinguish among the generations) is out of question: it would yield too heavy first generation masses. Thus we will need to use family symmetries in constructing the fermion mass terms. The simplest way is just to extend the discrete symmetries used for the stabile picture in the Higgs sector also to the fermion sector.

For the demonstration, we will use the Model 1 presented in Section 4, which is based on a $Z_{3} \otimes Z_{n}^{(1)} \otimes Z_{n}^{(2)}$ discrete symmetry. The discrete charge assignments are given in table 2. The Higgs sector is given by the superpotential of eq. 4.32. Because we have now two adjoint fields in one of the sectors of the accidental symmetry the $h_{1}, h_{2}$ Higgs doublet fields live in a linear combination of them. One can show that the uneaten PGB doublets are given by

$$
\begin{aligned}
& h_{1}=\cos \alpha\left(\cos \gamma h_{\Sigma_{1}}+\sin \gamma h_{\Sigma_{2}}\right)-\sin \alpha h_{H} \\
& h_{2}=\cos \alpha\left(\cos \gamma \bar{h}_{\Sigma_{1}}+\sin \gamma \bar{h}_{\Sigma_{2}}\right)-\sin \alpha \bar{h}_{\bar{H}}
\end{aligned}
$$


where $\tan \gamma=V_{2} / V_{1}$ and $\tan \alpha=3 V / U$. Here $\langle H\rangle=\langle\bar{H}\rangle=U,\left\langle\Sigma_{1,2}\right\rangle=V_{1,2}$, and $V=$ $\left(V_{1}^{2}+V_{2}^{2}\right)^{1 / 2}$.

If $V_{1} \sim V_{2} \ll U$, as it occurs e.g. for $n=6$, then the Higgs doublets are dominantly contained in $\Sigma_{1}$ and $\Sigma_{2}$ while almost not contained in $H$ and $\bar{H}$.

The allowed Yukawa couplings in this model together with their physical role are listed below.

- $15_{i} \bar{H} \overline{6}_{i}^{\prime}$ : makes the extra $\overline{5}$ 's and 5's heavy.

- 20H15 : makes the extra 10 and $\overline{10}$ heavy.

- 20 $\Sigma_{1} 20$ : yields heavy top quark.

- $\frac{1}{M^{2}} 20 \Sigma_{2} \bar{H} \bar{H} \bar{\sigma}_{3}$ : defines $\overline{6}_{3}$ state and yields b and $\tau$ masses.

- $\frac{1}{M^{2}} 20 \Sigma_{2} H 15_{2}$ : defines $15_{2}$ state and yields charm mass via c-t mixing.

- $\frac{1}{M^{2}} 15_{2,1} \Sigma_{1} \Sigma_{2} \bar{H} \overline{6}_{2}$ : gives s, $\mu$ masses and Cabbibo mixing.

- $\frac{1}{M^{4}} 15_{2,1} \Sigma_{1} \Sigma_{2} \bar{H}(\bar{H} H) \overline{6}_{3,1}$ : gives d,e masses and 1-3 mixing.

- $\frac{1}{M^{2}} 15_{2,1} H \Sigma_{1} \Sigma_{2} H 15_{2,1}$ : gives u mass.

M denotes the suppression scale of the nonrenormalizable operators. We denote $\epsilon_{H}=\frac{\langle H\rangle}{M}$, $\epsilon=\frac{\langle\Sigma\rangle}{\langle H\rangle}$. As we noted before, for $n=6\langle H\rangle$ is at an intermediate scale scale between $\langle\Sigma\rangle$ and $M_{P l}$. Since $\langle\Sigma\rangle=M_{G U T} \simeq 10^{16} \mathrm{GeV}$ is fixed (as the $S U(5)$ scale) by the gauge coupling unification, we obtain $\epsilon_{H} \sim \epsilon \sim 1 / 30$, which can explain the fermion mass hierarchy. A somewhat better fit can be obtained with the slightly larger value $\epsilon_{H} \sim 0.1$. This could occur if the above listed operators are generated by heavy fermion exchange (the heavy particles should be below the Planck scale, with masses $M \sim 10^{18} \mathrm{GeV}$ ). The desired value of the parameter $\epsilon$ should remain $\sim 1 / 30$ which fits perfectly to the light generation fermion masses.

The physical consequences of the above listed operators can be summarized as follows $(\lambda$ denotes the Yukawa couplings of the MSSM, while $\theta_{i j}$ denotes the mixing angle of the i'th and j'th generation):

$-\lambda_{t} \sim 1$

$-\lambda_{b, \tau} \sim \epsilon_{H}^{2}, \lambda_{b}=\lambda_{\tau}$.

$-\lambda_{c} \sim \epsilon_{H}^{2}$

$-\sin \theta_{23}=\sqrt{\frac{\lambda_{c}}{\lambda_{t}}} \sim \epsilon_{H}$.

- $\lambda_{s, \mu} \sim \epsilon \epsilon_{H}^{2}$, but the ratio $\lambda_{s} / \lambda_{\mu}$ is not fixed (because there is more than one operator due to the different possible contractions of indices in the operator $15_{2,1} \Sigma_{1} \Sigma_{2} \bar{H} \overline{6}_{2}$ ). 
- $\lambda_{d, e} \sim \epsilon \epsilon_{H}^{4}$, but the ratio of the couplings is not fixed again. The ratio $\lambda_{e} / \lambda_{\mu}$ is of order $\epsilon_{H}^{2} \sim \lambda_{c} / \lambda_{t}$. However $\lambda_{d}$ is a somewhat small.

- $\lambda_{u} \sim \epsilon \epsilon_{H}^{3}$. The ratio $\lambda_{u} / \lambda_{c}$ is of order $\epsilon \epsilon_{H} \sim \lambda_{\mu} / \lambda_{\tau} \sqrt{\lambda_{c} / \lambda_{t}}$.

$-\sin \theta_{12} \sim \mathcal{O}(1)$ (Cabbibo angle).

$-\sin \theta_{13} \sim \frac{\lambda_{d}}{\lambda_{b}}$.

All these consequences (except the down mass which must be enhanced by introducing a large Clebsch coefficient) are in qualitative agreement with the experimental values, provided that $\tan \beta$ is small (close to 1 ). In fact, here we used the general operator analysis consistent with the gauge $S U(6)$ and discrete symmetries. By addressing the specific heavy fermion exchanges, one could also fix the relative Clebsch factors between the down quark and charged lepton masses [13, 14]. Thus we have shown that a consistent model based on the $\mathrm{SU}(6)$ gauge group and discrete symmetries can be constructed. In this model the accidental global $\mathrm{SU}(6) \otimes \mathrm{SU}(6)$ symmetry is preserved by nonrenormalizable operators in the Higgs superpotential up to sufficiently high order terms, so that the Higgs doublets are PGB's without any fine tuning. On the other hand, Yukawa terms explicitly violating the $\mathrm{SU}(6) \otimes \mathrm{SU}(6)$ symmetry yield the necessary Yukawa couplings for the light fermion masses.

\section{References}

[1] E. Witten, Phys. Lett. B105 (1981), 287; D.V. Nanopoulos and K. Tamvakis, Phys. Lett. B113 (1982), 151.

[2] S. Dimopoulos and F. Wilczek, in Erice Summer Lectures, Plenum, New York, 1981; B. Grinstein, Nucl. Phys. B206 (1982), 387; H. Georgi, Phys. Lett. B108 (1982), 283; A. Masiero et al.,, Phys. Lett. B115 (1982) 380.

[3] I. Antoniadis, J. Ellis, J.S. Hagelin, D.V. Nanopoulos, Phys. Lett. B194 (1987), 231.

[4] H.P. Nilles, M. Srednicki and D. Wyler, Phys. Lett. B124 (1982) 337; A. Lahanas, ibid. 341; D. Nemeschansky, Nucl. Phys. B234 (1984), 379.

[5] S. Dimopoulos and F. Wilczek, NSF-ITP-82-07 (unpublished); M. Srednicki, Nucl. Phys. B202 (1982), 327.

[6] K.S. Babu and S.M. Barr, Phys. Rev. D48 (1993), 5354; Phys. Rev. D50 (1994), 3529. 
[7] K. Inoue, A. Kakuto and H. Takano, Prog. Theor. Phys. 75 (1986), 664.

[8] A. Anselm and A. Johansen, Phys. Lett. B200 (1988), 331;

A. Anselm, Sov. Phys. JETP 67 (1988), 663.

[9] R. Barbieri, G. Dvali, A. Strumia, Nucl. Phys. B391 (1993), 487.

[10] G.F. Guidice, A. Masiero, Phys. Lett. B206 (1988), 480.

[11] Z. Berezhiani and G. Dvali, Sov. Phys. Lebedev Inst. Rep. 5 (1989), 55.

[12] R. Barbieri, G. Dvali, M. Moretti, Phys. Lett. B312 (1993), 137.

[13] R. Barbieri, G. Dvali, A. Strumia, Z. Berezhiani, L. Hall, Nucl. Phys. B432 (1994), 49.

[14] R. Barbieri and Z. Berezhiani, INFN-FE 13-94; Z. Berezhiani, INFN-FE 14-94, hepph/9412372.

[15] R.C. King, Nucl. Phys. B185 (1981), 133.

[16] C.D. Frogatt and H.B. Nielsen, Nucl. Phys. B147 (1979), 277; Z. Berezhiani, Phys. Lett. B129 (1983) 99; B150 (1985) 177; S. Dimopoulos, Phys. Lett. B129 417; J. Bagger, S. Dimopoulos, H. Georgi and S. Raby, in Proc. Fifth Workshop on Grand Unification, eds. Q. Kang et al., World Scientific, Singapore, 1984. 